\title{
Nonmodal stability in Hagen-Poiseuille flow of a shear thinning fluid
}

\author{
Rong Liu* and Qiu Sheng Liu ${ }^{\dagger}$ \\ Key Laboratory of Microgravity (National Microgravity Laboratory), Institute of Mechanics, Chinese Academy of Sciences, \\ Beijing 100190, China
}

(Received 30 December 2011; revised manuscript received 24 April 2012; published 22 June 2012)

\begin{abstract}
Linear stability in Hagen-Poiseuille flow of a shear-thinning fluid is considered. The non-Newtonian viscosity is described by the Carreau rheological law. The effects of shear thinning on the stability are investigated using the energy method and the nonmodal stability theory. The energy analysis shows that the nonaxisymmetric disturbance with the azimuthal wave number $m=1$ has the lowest critical energy Reynolds number for both the Newtonian and shear-thinning cases. With the increase of shear thinning, the critical energy Reynolds number decreases for both the axisymmetric and nonaxisymmetric cases. For the nonmodal stability, we focus on two problems: response to external excitations and response to initial conditions. The former is studied by examining the $\epsilon$ pseudospectrum, and the latter by examining the energy growth function $G(t)$. For both Newtonian and shear-thinning fluids, it is found that there can be a rather large transient growth even though the linear operator of the Hagen-Poiseuille flow has no unstable eigenvalue. For the problem of response to external excitations, the optimal response is achieved by disturbance with $m=1$ for both the Newtonian and non-Newtonian cases. For the problem of response to initial conditions, the optimal disturbance is in the form of streamwise uniform streaks. Being different from the Newtonian case, the azimuthal wave number of the optimal disturbance may be greater than 1 for strongly shear-thinning cases.
\end{abstract}

DOI: 10.1103/PhysRevE.85.066318

PACS number(s): 47.50.Gj

\section{INTRODUCTION}

The transition from laminar to turbulent flow in a circular pipe is one of the most intriguing problems of classical hydrodynamics since the original work of Reynolds [1]. Since that time extensive experimental and theoretical investigations have been made. However, an understanding of the mechanism of transition from laminar towards turbulence is far from complete.

Transition to turbulence in a circular pipe flow of a Newtonian fluid is governed by one single dimensionless parameter, the Reynolds number $\operatorname{Re}=U R / v$, where $U$ is the mean flow speed along the centerline of the pipe, $R$ the radius, and $v$ is the kinematic viscosity of the fluid. It is well known that the Poiseuille flow in a circular pipe (Hagen-Poiseuille flow) is linear stable, i.e., no critical Reynolds number exists above which disturbances grow exponentially [2,3]. However, in most experiments transition in a circular pipe flow is observed at $\operatorname{Re} \approx 2000$ [4], whereas, in more tightly controlled conditions, laminar flow can be maintained until $\mathrm{Re} \approx 12000$. A more precise value above which transition to turbulence can be triggered is difficult to identify. Subsequent experimental works have essentially confirmed the lowest critical value ranging between 1760 and 2300 [5]. For more previous works on the transition from laminar to turbulence in a circular pipe, we refer the reader to the review articles $[5,6]$ and references therein.

Classical linear hydrodynamic stability analysis is also found to give misleading results even for other flows, such as simple Couette and Poiseuille flows. Because of the discrepancies between linear stability analysis and experiments,

\footnotetext{
*liurong@imech.ac.cn

†liu@imech.ac.cn
}

transition in shear flows must be explained in terms of other mechanisms. Until now, no full theory of transition in shear flows exists. Nevertheless significant progress has been achieved due to the emergence of the nonmodal stability theory. Traditionally, a first step in investigating transition is the normal-mode analysis. For this approach, it is assumed that each mode has an exponential time dependence, thus the base flow is considered to be unstable if an eigenvalue is found in the unstable complex half plane. For linear stability problem of shear flows, the operator is highly non-normal. The spectrum is a poor proxy for the disturbance behavior as it only describes the asymptotic fact of the perturbation and fails to capture short-term characteristics. The main point of departure of the nonmodal theory from the traditional eigenvalue analysis is the fact that even if all of the eigenvalues of a linear system are distinct and lie well inside the stable half plane, inputs to that system may be amplified by substantially large factors if the linear operator is non-normal. In the nonmodal theory, stability is redefined in a broader sense as the response behavior of the governing equations to general input variables in the form of initial conditions, external forcing, and internal uncertainties [7].

Trefethen et al. [8] addressed the general concept of nonmodal stability analysis and studied the response to external excitations and initial conditions for plane Couette and Poiseuille flows in the framework of nonmodal theory. Reddy and Henningson [9] have studied the transient energy growth in viscous channel flow. Schimid and Henningson [2] have studied the optimal energy density growth in Hagen-Poiseuille flow using nonmodal approach. The common conclusion of the above studies is that disturbance may experience a substantial amplification even for exponentially damped modes because of the transient growth mechanism [10]. This mechanism is primarily due to linear amplification of disturbances energized by the background mean shear [8,11]. It occurs in the absence 
of nonlinear effects, and bypasses the primary (secondary) instabilities.

For inviscid shear flows, Ellingsen and Palm [12] showed that the streamwise velocity component can increase linearly with time for disturbance with no streamwise variation, producing alternating low and high velocity streaks in the streamwise velocity component. In laminar shear flows, it is known that streaks have the potential to be largely amplified from streamwise vortices through the "lift-up" effect $[13,14]$. This mechanism works by efficiently extracting momentum from the mean flow and transferring it to the perturbation. Mathematically speaking, the nonmodal theory offers an explanation of why these streamwise streaks are so common: even though these structures are not eigenmodes of the linearized flow problem, they are pseudomodes [8]. Reddy and Henningson [9] computed the optimally amplified disturbances in channel flows using a singular value analysis. It is found that initial states with largest transient energy growth in subcritical flows lead to flow structures that resemble streamwise vortices and streaks.

Given the apparently potent linear amplification of the optimal disturbances predicted by nonmodal theory, interest was naturally focused on examining how energy could be fed back from the streaks into the secularly decaying rolls so that the process could be sustainable and thereby accomplish transition. Waleffe [15] proposed the self-sustaining process (SSP) to understand how turbulence is maintained rather than initiated at low Reynolds numbers. As is well known, the SSP consists of three distinct phases: formation of streamwise streaky flow, instability of streaky flow, and nonlinear feedback on the rolls. The mechanism of the transient growth is responsible for the formation of streaks. In the present paper, the nonmodal theory is used to investigate the formation of streaks in the linear stage.

Shear flows of non-Newtonian fluids are very common in industrial applications as well as in natural processes. In many flow applications, preventing a transition from laminar flow to turbulence is highly desirable. For many of these applications, understanding the influence of non-Newtonian effect on the instability of shear flows can be of critical importance. The non-Newtonian fluids have complex structures, including polymer solutions, polymer melts, soap solutions, suspensions, emulsions, and some biological fluids [16].

The physical mechanisms of non-Newtonian fluids are complex; here we focus on polymeric liquids. Because polymeric liquids contain high-molecular-weight molecules with many internal degrees of freedom, polymer solutions and molten polymers have behaviors qualitatively different from that of Newtonian fluids. One of the most important characteristics of macromolecular fluids is the non-Newtonian viscosity, i.e., the fact that the viscosity $\mu$ changes with the shear rate $\dot{\gamma}$. A shear-thinning (shear-thickening) fluid, also called a pseudoplastic (dilatant) fluid, is a non-Newtonian fluid where the shear viscosity decreases (increases) with applied shear stress. Shear-thinning fluids are usually solutions of large, polymeric molecules in a solvent with smaller molecules. Most macromolecular fluids are shear thinning, and shear-thickening fluids are rarely encountered. The generalized Newtonian model has been widely used to describe the shear rate dependence of the viscosity. Various models, such as the power law mode and the Carreau model, can be used to describe the relation between viscosity and shear rate [17].

Although shear-thinning fluids are commonly used in industrial process, there is surprisingly little published work that focuses on transient behavior of these flows. Two recent papers $[18,19]$ have examined the influences of shear-thinning effect on the transient growth of a plane Poiseuille flow. Chikkadi et al. [18] have studied two typical cases of channel flow. One is the plane Poiseuille flow of a Carreau fluid, the other is two miscible fluids of equal densities but different viscosities. Rather unexpectedly, the authors found that a stratification of viscosity alone does not affect transient growth; moreover, the transient growth is only slightly influenced by the shear-thinning effect. The result seems quite important to the requirement of preventing transition to turbulent. However, we should note that the results are only valid for neglecting the viscosity perturbations. Nouar et al. [19] have revisited the stability of shear-thinning fluids. Being different from the results in [18], the authors have accounted for a nonvanishing viscosity disturbance, and found that shear thinning appears to be destabilizing when Re is based on the average effective viscosity. More recently, Liu and Liu [20] have studied the nonmodal stability in plane Couette flow of a power-law fluid. The above works are limited to the plane shear flows. However, a careful look at previous works indicates that investigation on the effect of non-Newtonian viscosity on transient behavior in Hagen-Poiseuille flow is very limited. Recently, Esmael and Nouar [21] studied the transitional flow of a yield-stress fluid in a pipe flow. The experimental result in Ref. [21] indicated that a robust nonlinear coherent structure mediating the transition from laminar to turbulent flow exists. This coherent structure characterized by two counter-rotating longitudinal vortices is similar to the optimal disturbance obtained by the nonmodal analysis. In the present paper, we consider the problem of transition from laminar flow to turbulence in Hagen-Poiseuille flow of a shear-thinning fluid. The purpose is to understand the effect of shear thinning on the nonmodal behaviors, including responses to excitations and transient energy growth of initial conditions in Hagen-Poiseuille flow.

The paper is organized as follows. In Sec. II, the mathematical formulation of the physical model is presented. In Sec. III, the background of the nonmodal theory is briefly described. The numerical method is presented in Sec. IV. In Sec. V, we present the results and discussions. Finally, in Sec. VI, we summarize the results and present the conclusions.

\section{MATHEMATICAL FORMULATION}

The motion of an incompressible, shear-thinning fluid in a circular pipe of radius $R$ is considered. The flow is driven by a constant pressure gradient along the axis of the pipe. The dimensionless Navier-Stokes equations are expressed as

$$
\begin{gathered}
\nabla \cdot \boldsymbol{u}=0, \\
\frac{\partial \boldsymbol{u}}{\partial t}+\boldsymbol{u} \cdot \nabla \boldsymbol{u}=-\nabla p+\nabla \cdot \boldsymbol{\tau},
\end{gathered}
$$


(a)

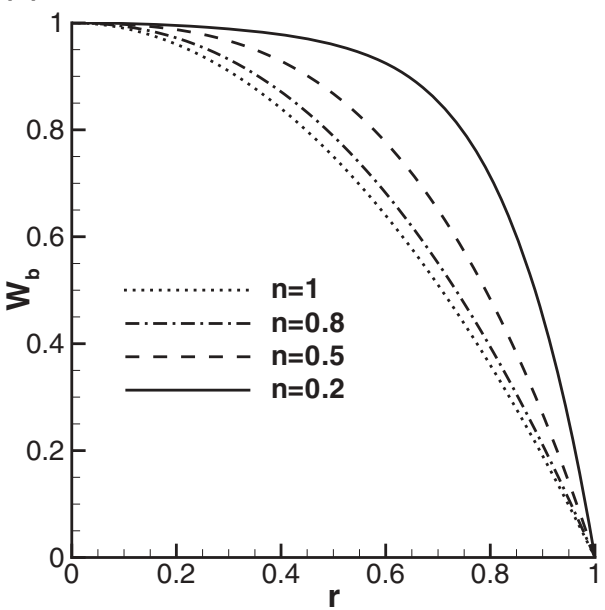

(b)

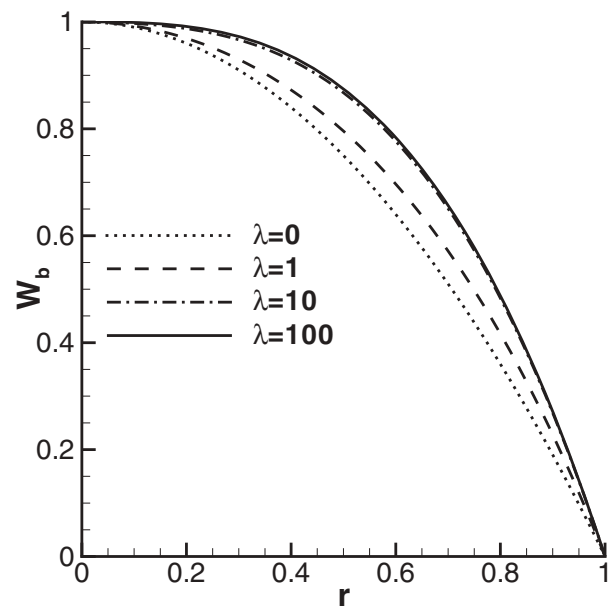

FIG. 1. Velocity profiles for base state in the case of $\hat{\mu}_{\infty}=0$. (a) Effect of $n$ at $\lambda=10$; (b) effect of $\lambda$ at $n=0.5$.

where $p$ is the pressure, $\boldsymbol{\tau}$ is the stress tensor, and $\boldsymbol{u}=u \mathbf{e}_{r}+$ $v \mathbf{e}_{\theta}+w \mathbf{e}_{z}$ is the velocity vector, in which $\mathbf{e}_{r}, \mathbf{e}_{\theta}$, and $\mathbf{e}_{z}$ are the unit vectors in the radial, azimuthal, and axial directions. In these equations, the variables have been normalized by selecting $R, U_{c} / R, U_{c}$, and $\rho U_{c}^{2}$ as the length, time, velocity, and pressure scales. Here $\rho$ is the density of fluid and $U_{c}$ denotes the base velocity at the centerline.

In the present study, we choose the Carreau model to describe the viscosity $\mu$. We define the rate of strain tensor as $\dot{\gamma}=\nabla \boldsymbol{u}+(\nabla \boldsymbol{u})^{T}$, and the magnitude of the rate of strain tensor as $\dot{\gamma}=\sqrt{\frac{1}{2}(\dot{\gamma}: \dot{\gamma})}$. The constitutive relation is

$$
\mu=\frac{\hat{\mu}_{\infty}}{\hat{\mu}_{0}}+\left(1-\frac{\hat{\mu}_{\infty}}{\hat{\mu}_{0}}\right)\left[1+(\lambda \dot{\gamma})^{2}\right]^{(n-1) / 2},
$$

in which the power-law index $n$ represents the degree of shear thinning, $\lambda$ is the time constant of the fluid, $\hat{\mu}_{0}$ is the zeroshear-rate viscosity, and $\hat{\mu}_{\infty}$ is the infinite-shear-rate viscosity. The infinite-shear-rate viscosity $\hat{\mu}_{\infty}$ is frequently significantly smaller $\left(10^{-3}-10^{-4}\right.$ times $)$ than $\hat{\mu}_{0}$. For $n=1$ or $\lambda=0$, the viscosity reduces to the non-Newtonian case. The expression for the stress tensor is given by

$$
\boldsymbol{\tau}=\frac{1}{\mathrm{Re}} \mu(\dot{\gamma}) \dot{\gamma}
$$

in which Re is the Reynolds number defined as

$$
\operatorname{Re}=\frac{\rho R U_{c}}{\hat{\mu}_{0}} .
$$

\section{A. Basic flow}

Before we study the linear stability of the system, we need to find the steady solution of the unidirectional base flow. In the base state, the velocity components and the magnitude of $\dot{\gamma}$ are

$$
\overline{\boldsymbol{u}}=\left(0,0, W_{b}(r)\right), \quad \overline{\dot{\gamma}}=\left|\overline{\dot{\gamma}}_{z r}\right|=\left|\frac{d}{d r} W_{b}(r)\right|,
$$

in which $W_{b}(r)$ denotes the axial component of the velocity of base state. Suppose $d \bar{p} / d z<0$; the base flow satisfies

$$
\frac{\operatorname{Re}}{2} \frac{d \bar{p}}{d z} r=\mu(\overline{\dot{\gamma}}) \overline{\dot{\gamma}}_{r z} .
$$

We need to solve this nonlinear equation for $\overline{\dot{\gamma}}_{r z}$ by iterative method. Once we have found the value of $\overline{\dot{\gamma}}$, the base velocity can be obtained from

$$
\frac{d}{d r} W_{b}=\overline{\dot{\gamma}}_{r z}
$$

together with the boundary condition of $W_{b}(1)=0$. Note that we need to vary the parameter $\operatorname{Re}(d p / d z)$, so that the base flow satisfies $W_{b}(0)=1$. We do this by using the bisection method.

The axial velocity profiles of the base state are plotted in Fig. 1 for several typical parameters. In Fig. 1(a), the effect of index $n$ on the velocity profile of the base state is presented for $\lambda=10$. In this figure, with the increase of shear thinning the shear rate increases near the wall and the profile flattens near the center line of the pipe. In Fig. 1(b), the effect of time constant $\lambda$ on the velocity profile of the base state is presented for $n=0.5$. For small values of $\lambda$, with the increase of $\lambda$ the shear rate increases near the wall and the profile flattens near the center line of the pipe. However, for large values of $\lambda$ the profile is insensitive to $\lambda$.

\section{B. Linearized equations}

Our starting point for the analysis of infinitesimal disturbances $\left(\boldsymbol{u}^{\prime}, p^{\prime}\right)$ in circular pipe flow is to linearize the Navier-Stokes equation around $(\overline{\boldsymbol{u}}, \bar{p})$ :

$$
\nabla \cdot \boldsymbol{u}^{\prime}=0
$$

$$
\frac{\partial \boldsymbol{u}^{\prime}}{\partial t}+\overline{\boldsymbol{u}} \cdot \nabla \boldsymbol{u}^{\prime}+\boldsymbol{u}^{\prime} \cdot \nabla \overline{\boldsymbol{u}}=-\nabla p^{\prime}+\nabla \cdot \boldsymbol{\tau}^{\prime},
$$

where $\boldsymbol{\tau}^{\prime}$ is the shear stress perturbation given by $\boldsymbol{\tau}^{\prime}=\left(\bar{\mu} \dot{\boldsymbol{\gamma}}^{\prime}+\right.$ $\left.\mu^{\prime} \overline{\dot{\gamma}}\right) /$ Re. The viscosity perturbation $\mu^{\prime}$ can be expressed as

$$
\mu^{\prime}=\left.\frac{d \mu}{d \dot{\gamma}}\right|_{\boldsymbol{u}=\overline{\boldsymbol{u}}} \dot{\gamma}^{\prime}=\left.\frac{d \mu}{d \dot{\gamma}}\right|_{\boldsymbol{u}=\overline{\boldsymbol{u}}} \operatorname{sgn}\left(\overline{\dot{\gamma}}_{z r}\right) \dot{\gamma}_{z r}^{\prime} .
$$


It can be shown straightforwardly that

$$
\begin{array}{ccc}
\tau_{i j}^{\prime}=\bar{\mu} \dot{\gamma}_{i, j}^{\prime} & \text { for } \quad i j \neq r z, z r, \\
\tau_{i j}^{\prime}=\mu_{t} \dot{\gamma}_{i, j}^{\prime} & \text { for } \quad i j=r z, z,
\end{array}
$$

in which $\mu_{t}$ is the tangent viscosity defined as $\mu_{t}=\bar{\mu}+$ $\left.\frac{d \mu}{d \dot{\gamma}}\right|_{\boldsymbol{u}=\overline{\boldsymbol{u}}} \overline{\dot{\gamma}}$. For a non-Newtonian fluid, the difference between $\mu_{t}$ and $\bar{\mu}$ identifies the departure from Newtonian viscosity. For a Carreau fluid, $\mu_{t}-\bar{\mu}=(n-1)\left(1-\hat{\mu}_{\infty} / \hat{\mu}_{0}\right)[1+$ $\left.(\lambda \dot{\gamma})^{2}\right]^{(n-3) / 2}(\lambda \dot{\gamma})^{2}$. At last we obtain the perturbed stress tensor as

$$
\begin{aligned}
\nabla \cdot \boldsymbol{\tau}^{\prime}= & \frac{1}{\operatorname{Re}}\left\{\bar{\mu} \nabla^{2} \boldsymbol{u}^{\prime}+\frac{d \bar{\mu}}{d r} \mathbf{e}_{r} \cdot \dot{\boldsymbol{\gamma}}^{\prime}+\left(\mu_{t}-\bar{\mu}\right) \frac{\partial \dot{\gamma}_{z r}^{\prime}}{\partial z} \mathbf{e}_{r}\right. \\
& \left.+\frac{1}{r} \frac{\partial r\left(\mu_{t}-\bar{\mu}\right) \dot{\gamma}_{r z}^{\prime}}{\partial r} \mathbf{e}_{z}\right\} .
\end{aligned}
$$

For the remainder of the present paper, we omit the prime of all variables for simplicity. The linearized equations are expressed in component form in cylindrical coordinates:

$$
\begin{gathered}
\frac{\partial u}{\partial r}+\frac{u}{r}+\frac{1}{r} \frac{\partial v}{\partial \theta}+\frac{\partial w}{\partial z}=0, \\
\frac{\partial u}{\partial t}+W_{b} \frac{\partial u}{\partial z}=-\frac{\partial p}{\partial r}+\frac{1}{\operatorname{Re}}\left\{\bar{\mu}\left[\Delta u-\frac{u}{r^{2}}-\frac{2}{r^{2}} \frac{\partial v}{\partial \theta}\right]\right. \\
\left.+2 \frac{d \bar{\mu}}{d r} \frac{\partial u}{\partial r}+\left(\mu_{t}-\bar{\mu}\right) \frac{\partial}{\partial z}\left(\frac{\partial w}{\partial r}+\frac{\partial u}{\partial z}\right)\right\}, \\
\frac{\partial v}{\partial t}+W_{b} \frac{\partial v}{\partial z}=-\frac{1}{r} \frac{\partial p}{\partial \theta}+\frac{1}{\operatorname{Re}}\left\{\bar{\mu}\left[\Delta v-\frac{v}{r^{2}}+\frac{2}{r^{2}} \frac{\partial u}{\partial \theta}\right]\right. \\
\left.+\frac{d \bar{\mu}}{d r}\left(\frac{1}{r} \frac{\partial u}{\partial \theta}+\frac{\partial v}{\partial r}-\frac{v}{r}\right)\right\}, \\
\frac{\partial w}{\partial t}+W_{b} \frac{\partial w}{\partial z}+\frac{d W_{b}}{d r} u \\
-\frac{\partial p}{\partial z}+\frac{1}{\operatorname{Re}}\left\{\bar{\mu} \Delta w+\frac{d \bar{\mu}}{d r}\left(\frac{\partial w}{\partial r}+\frac{\partial u}{\partial z}\right)\right. \\
+\frac{d\left(\mu_{t}-\bar{\mu}\right)}{d r}\left(\frac{\partial w}{\partial r}+\frac{\partial u}{\partial z}\right) \\
\left.+\left(\mu_{t}-\bar{\mu}\right)\left[\frac{1}{r}\left(\frac{\partial w}{\partial r}+\frac{\partial u}{\partial z}\right)+\frac{\partial}{\partial r}\left(\frac{\partial w}{\partial r}+\frac{\partial u}{\partial z}\right)\right]\right\}
\end{gathered}
$$

in which

$$
\Delta=\frac{\partial^{2}}{\partial r^{2}}+\frac{1}{r} \frac{\partial}{\partial r}+\frac{1}{r^{2}} \frac{\partial^{2}}{\partial \theta^{2}}+\frac{\partial^{2}}{\partial z^{2}} .
$$

As the flow is periodic in the azimuthal direction and will be assumed periodic in the streamwise direction, all solutions to the linearized controlling equations can be expressed as superpositions of complex Fourier modes of the form

$$
\boldsymbol{u}(r, \theta, z, t)=\hat{\boldsymbol{u}}(r, t) e^{i(m \theta+k z)}, \quad p(r, \theta, z, t)=\hat{p}(r, t) e^{i(m \theta+k z)},
$$

in which the streamwise wave number $k \in \mathscr{R}$, and the azimuthal wave number $m \in \mathscr{Z}$.
At last we obtain the normal mode equations for disturbances as

$$
D \hat{u}+\frac{\hat{u}}{r}+\frac{i m}{r} \hat{v}+i k \hat{w}=0,
$$

$$
\begin{aligned}
\partial_{t} \hat{u}= & -D \hat{p}-i k W_{b} \hat{u}+\frac{\bar{\mu}}{\operatorname{Re}}\left[\left(D^{2}+\frac{D}{r}\right) \hat{u}\right. \\
& \left.-\left(\frac{m^{2}+1}{r^{2}}+k^{2}\right) \hat{u}-\frac{2 i m}{r^{2}} \hat{v}\right] \\
& +\frac{1}{\operatorname{Re}}\left[2 D \bar{\mu} D \hat{u}+\left(\mu_{t}-\bar{\mu}\right)\left(i k D \hat{w}-k^{2} \hat{u}\right)\right],
\end{aligned}
$$

$$
\begin{aligned}
\partial_{t} \hat{v}= & -\frac{i n}{r} \hat{p}-i k W_{b} \hat{v}+\frac{\bar{\mu}}{\operatorname{Re}}\left[\left(D^{2}+\frac{D}{r}\right) \hat{v}\right. \\
& \left.-\left(\frac{m^{2}+1}{r^{2}}+k^{2}\right) \hat{v}+\frac{2 i m}{r^{2}} \hat{u}\right] \\
& +\frac{D \bar{\mu}}{\operatorname{Re}}\left[\frac{i m}{r} \hat{u}+D \hat{v}-\frac{\hat{v}}{r}\right],
\end{aligned}
$$

$$
\partial_{t} \hat{w}=-i k \hat{p}-i k W_{b} \hat{w}-\hat{u} D W_{b}
$$

$$
\begin{aligned}
& +\frac{\bar{\mu}}{\operatorname{Re}}\left[\left(D^{2}+\frac{D}{r}\right) \hat{w}-\left(\frac{m^{2}}{r^{2}}+k^{2}\right) \hat{w}\right] \\
& +\frac{1}{\operatorname{Re}}\left[\left(D\left(\mu_{t}-\bar{\mu}\right)+\frac{\mu_{t}-\bar{\mu}}{r}\right)(D \hat{w}+i k \hat{u})\right. \\
& \left.+\left(\mu_{t}-\bar{\mu}\right)\left(D^{2} \hat{w}+i k D \hat{u}\right)\right]
\end{aligned}
$$

in which $D \equiv \partial / \partial r$.

The boundary conditions at $r=1$ are given by the no-slip assumption and can be written in the form

$$
\hat{u}=\hat{v}=\hat{w}=0 .
$$

The center line of $r=0$ is a regular singular point. The regularity conditions will be determined by the boundedness of the solution $(\hat{\boldsymbol{u}}, \hat{p})$. The regularity conditions can be obtained by considering the Taylor expansion of the fields $(\hat{\boldsymbol{u}}, \hat{p})$ as functions of $r$ [22]. A more straightforward way to obtain the conditions at $r=0$ is to use the fact that the velocity vector has a vanishing azimuthal dependence as the center line is approached [23]. That is,

$$
\lim _{r \rightarrow 0} \frac{\partial \boldsymbol{u}}{\partial \theta}=\lim _{r \rightarrow 0} \frac{\partial p}{\partial \theta}=0 .
$$

Note that

$$
\begin{aligned}
\lim _{r \rightarrow 0} \frac{\partial \boldsymbol{u}}{\partial \theta} & =\frac{\partial u}{\partial \theta} \mathbf{e}_{r}+\frac{\partial v}{\partial \theta} \mathbf{e}_{\theta}+\frac{\partial w}{\partial \theta} \mathbf{e}_{z}+u \frac{d \mathbf{e}_{r}}{d \theta}+v \frac{d \mathbf{e}_{\theta}}{d \theta}+w \frac{d \mathbf{e}_{z}}{d \theta} \\
& =\left(\frac{\partial u}{\partial \theta}-v\right) \mathbf{e}_{r}+\left(\frac{\partial v}{\partial \theta}+u\right) \mathbf{e}_{\theta}+\frac{\partial w}{\partial \theta} \mathbf{e}_{z} .
\end{aligned}
$$

Thus regular conditions at the centerline are

$$
\frac{\partial u}{\partial \theta}-v=\frac{\partial v}{\partial \theta}+u=\frac{\partial w}{\partial \theta}=\frac{\partial p}{\partial \theta}=0 ;
$$

in Fourier mode they are expressed as

$$
i m \hat{u}-\hat{v}=i m \hat{v}+\hat{u}=m \hat{w}=m \hat{p}=0 .
$$

For $m=0$,

$$
\hat{u}=\hat{v}=0, \quad D \hat{w}=D \hat{p}=0 .
$$


For $m= \pm 1$,

$$
D \hat{u}=D \hat{v}=0, \quad \hat{w}=0, \quad \hat{p}=0 .
$$

For $|m| \geqslant 2$,

$$
\hat{u}=\hat{v}=\hat{w}=\hat{p}=0 .
$$

\section{TRANSIENT BEHAVIORS AND NONMODAL INSTABILITIES}

In the framework of nonmodal theory, two types of problems are of particular interest, i.e., the responses to external excitations and the transient energy growth of initial conditions. For the former, the norm of resolvent denotes the maximum amplification of external excitations; for the latter, the growth function $G(t)$ identifies the optimal growth of energy at time $t$.

We begin with the transient behavior of responses to external excitations for a general linear system $\frac{d}{d t} \mathbf{U}=-i \mathscr{L} \mathbf{U}$. Suppose the system is driven by a harmonic signal in the form of

$$
\mathbf{V}(r, \theta, z, t)=e^{i(-\omega t+k z+m \theta)} \mathbf{v}(r) .
$$

Assuming that system is stable, the response is

$$
\mathbf{U}(r, \theta, z, t)=i e^{i(-\omega t+k z+m \theta)}(\omega \mathscr{I}-\mathscr{L})^{-1} \mathbf{v}(z) .
$$

Here $\mathscr{I}$ is the identity matrix. The solution operator $(\omega \mathscr{I}-$ $\mathscr{L})^{-1}$ is known as the resolvent. The maximum value of amplification is equal to the norm of the resolvent,

$$
\mathcal{R}(k, m, \omega)=\max _{\mathbf{v} \neq 0} \frac{\|\mathbf{U}\|}{\|\mathbf{V}\|}=\left\|(\omega \mathscr{I}-\mathscr{L})^{-1}\right\| .
$$

Here $\|\cdot\|$ denotes a norm on $\mathbb{C}^{N}$, max denotes maximum. An eigenvalue of $\mathscr{L}$ is a number $\omega$ such that $\|(\omega \mathscr{I}-$ $\mathscr{L})^{-1} \| \rightarrow \infty$. Generalizing this result leads naturally to the definition of " $\epsilon$ pseudospectra" [24]. In mathematical meaning, the resolvent norm represents the the amplification of responses to external forcing. So, examining the structure of the pseudospectra is helpful to understand the influence of shear-thinning effect on the nonmodal stability of pipe Poiseuille flow. In the present paper, when investigating the responses to external forcing, we fix the parameters including the streamwise and azimuthal wave numbers and the Reynolds number, and plot the eigenvalues, the isolines of $\epsilon$ pseudospectra, and the numerical range. The value of $\epsilon^{-1}$ corresponds to the maximal response $\mathcal{R}(k, m, \omega)$.

The quest for maximum amplification of initial conditions is of particular interest in many hydrodynamic stability problems. For the linear system, the solution has the form of

$$
\mathbf{u}(t)=\exp (-i \mathscr{L} t) \mathbf{u}(0) .
$$

The maximum amplification of the initial condition can be described by the growth function $G(t)$ as

$$
G(t)=\max _{q \neq 0} \frac{\|\mathbf{u}(t)\|^{2}}{\|\mathbf{u}(0)\|^{2}}=\left\|e^{-i \mathscr{L} t}\right\|^{2} .
$$

We should note that $\mathcal{R}(\omega)$ and $G(t)$ are related to the choice of definition of the norm $\|\cdot\|$. In the present paper, we choose the norm $\|\cdot\|$ as the energy norm.

For initial value problems in hydrodynamics, we may be interested in the energy growth rate at the initial stage. The concept of numerical range can be used to link the operator to the initial energy growth. The numerical range is defined as

$$
W(\mathbf{A})=\left\{\mathbf{x}^{*} \mathbf{A} \mathbf{x}: \mathbf{x} \in \mathbb{C}^{N},\|\mathbf{x}\|=1\right\},
$$

in which $\mathbf{A} \in \mathbb{C}^{N \times N}$. The numerical abscissa of $\mathbf{A}$ is defined as

$$
\omega(\mathbf{A})=\sup _{z \in W(\mathbf{A})} \operatorname{Re}(z)=\lim _{t \rightarrow 0} \frac{d}{d t}\left\|e^{\mathbf{A} t}\right\| .
$$

The main application of numerical range is to the analysis of energy growth for initial value problems. The numerical abscissa of $-i \mathscr{L}$ corresponds to the initial growth rate of the energy growth function $[9,24]$.

\section{NUMERICAL METHOD}

In the present paper, we will examine the nonmodal stability, including the response to external excitations and initial conditions. We need to compute the eigenvalues and eigenvectors of the operator $\mathscr{L}$, the pseudospectrum, the numerical range, and the energy growth function $G(t)$. The spectral method can yield great accuracy for hydrodynamic stability problems. In the present paper, a Chebyshev-collocation method is used to solve the eigenvalue problem, and an eigenmode expansion method is used to solve the nonmodal problem.

We begin with the computation of eigenvalue and eigenvectors. We first transform the domains of $r \in[0,1]$ to the Chebyshev domain $\zeta \in[-1,1]$ by introducing

$$
\zeta=2 r-1 \text {. }
$$

A Chebyshev collocation method is implemented to solve the eigenvalue problem. In this approach, the variables $\hat{\boldsymbol{u}}$ and $\hat{p}$ are expanded as

$$
\hat{\boldsymbol{u}}=\sum_{n=0}^{N} \hat{\boldsymbol{u}}_{n} T_{n}(\zeta), \hat{p}=\sum_{n=0}^{N} \hat{p}_{n} T_{n}(\zeta),
$$

in which $T_{n}$ denotes the $n$th Chebyshev polynomial.

Using the Chebyshev series (40) and substituting $\partial / \partial t$ with $-i \omega$, the governing equations can be written in discrete form

$$
\mathcal{A} \mathbf{x}=\omega \mathcal{B} \mathbf{x}
$$

in which $\mathbf{x}$ is the vector consisting of the spectral coefficients of $\hat{\boldsymbol{u}}$ and $\hat{p}$, and $\mathcal{A}, \mathcal{B}$ arise from discretization of the controlling equations and boundary conditions. This system of equations is required to solve for $4 N+4$ unknowns. The numerical method for the general eigenvalue problem in the form $\mathcal{A} \mathbf{x}=\omega \mathcal{B} \mathbf{x}$ has been described by Canuto et al. [25] and Schmid and Henningson [10]. For more details on the stability problem in pipe flows, we refer the reader to the works by Schimid and Henningson [2] and by Khorrammi et al. [23]. After we solve the eigenvalue problem, an eigenmode expansion method can be used to compute the transient energy growth and the response to external excitations. The computational method 
TABLE I. Relation between $\operatorname{Re}$ and $\overline{\operatorname{Re}}$ for various $n$ at $\lambda=10$.

\begin{tabular}{|c|c|c|c|c|c|c|c|c|c|c|}
\hline$n$ & 1.0 & 0.9 & 0.8 & 0.7 & 0.6 & 0.5 & 0.4 & 0.3 & 0.2 & 0.1 \\
\hline $\mathrm{Re} / \overline{\mathrm{Re}}$ & 1.0 & 0.7795 & 0.6105 & 0.4826 & 0.3883 & 0.3222 & 0.2820 & 0.2686 & 0.2899 & 0.3720 \\
\hline
\end{tabular}

is a standard procedure, and we refer the reader to Refs. [2] and [9].

\section{RESULTS AND DISCUSSIONS}

To assess the effect of shear thinning on the instability of pipe flows, it is important employ an appropriate viscosity scale in the definition of the Reynolds number. In computation, we use $\hat{\mu}_{0}$ as the viscosity scale. In the discussion of results, it is more appropriate to use the average viscosity or the tangent viscosity at the wall as the viscosity scale [19]. In the discussions, $\operatorname{Re}$ is converted to $\overline{\operatorname{Re}}$ in which the viscosity scale is the viscosity averaged across the pipe. We can convert Re to $\overline{\operatorname{Re}}$ by using $\operatorname{Re} / \overline{\operatorname{Re}}=\langle\hat{\mu}\rangle / \hat{\mu}_{0}$, in which $\langle$.$\rangle denotes averaged$ value across the pipe. The ratios of $\mathrm{Re}$ and $\overline{\mathrm{Re}}$ are listed in Table I for various non-Newtonian indexes $n$ at $\lambda=10$. As shown in Table I, the ratio of $\mathrm{Re} / \overline{\mathrm{Re}}$ is always less than unity for shear-thinning cases and at $n=0.3$ it has the minimum value. For $n \geqslant 0.3$ this ratio increases with the shear thinning, and for $n<0.3$ this value decreases with the shear thinning.

\section{A. Condition for no energy growth}

In this subsection, we are interested in the condition for which there is no growth of the perturbation kinetic energy. Energy methods give conditions for no energy growth. For a linear system $\frac{d}{d t} \mathbf{x}=-i \mathscr{L} \mathbf{x}$, we define the energy density $\mathscr{E}=\|\mathbf{x}\|_{2}^{2}$. There is no energy growth if and only if all the eigenvalues of the antisymmetric part of $\mathscr{L}$, i.e., $\frac{1}{2}\left(\mathscr{L}-\mathscr{L}^{H}\right)$, in which $H$ denotes the Hermit transpose, lies in the lower half plane. For hydrodynamics stability problems, the linear stability theory gives the sufficient conditions for instability, and the energy theory gives the sufficient conditions for stability. The energy method shows that there is no energy growth if the Reynolds number is less than $\mathrm{Re}_{g}$. In order to determine the critical energy Reynolds number $\mathrm{Re}_{g}$, two methods can be chosen. One way is to compute the eigenvalues of $\frac{1}{2}\left(\mathscr{L}-\mathscr{L}^{H}\right)$, and the other is to solve the optimization problem using a variational method. Reddy and Henningson [9] used the variational method to studied the energy stability of plane Couette and Poiseuille flow. In the present paper, we used the former one to study the energy stability of the problem.

We now examine the condition below which the kinetic energy of an infinitesimal disturbance decays monotonically. Let $\sigma$ be the leading eigenvalue of $\frac{1}{2}\left(\mathscr{L}-\mathscr{L}^{H}\right)$. In the $(k, \overline{\mathrm{Re}})$ plane, the condition with no energy growth is given by the energy Reynolds number $\overline{\operatorname{Re}}_{g}=\operatorname{Re}$ such that $\sigma=0$. Figure 2 present the boundary with no energy growth for both axisymmetric and nonaxisymmetric disturbances for various degrees of shear thinning. In Fig. 2(a) for $n=1$, the flow reduces to the Newtonian case. Compare the results in Fig. 2(a) with those in previous works; it is found that our compuational results are in good agreement with those in
Ref. [2]. In Fig. 2, it is shown that the structures of the curves for shear-thinning fluid are qualitatively similar to these for the Newtonian case. At all azimuthal wave numbers, the curves of the axisymmetric case of $m=0$ is always the most stable. For the nonaxisymmetric cases with $m>1$, the lowest Reynolds number is achieved at $k=0$. The global lowest energy Reynolds number is associated with nonaxisymmetric disturbances with the azimuthal wave number $m=1$ and the streamwsie wave number $k$ in the range $1-2$. Comparing the curves with different non-Newtonian index $n$, it is found that the energy Reynolds number decreases with the increase of shear thinning effect. For example, the global lowest energy number decreases from about 80 to about 40 as $n$ decreases from 1 to 0.3 .

\section{B. Transient behaviors and nonmodal stabilities}

In the nonmodal theory, two types of problems are of particular interest, i.e., response to external excitations and transient energy growth of initial conditions. For the former, the norm of resolvent denotes the maximum amplification of external excitations; for the latter, the growth function $G(t)$ identifies the optimal growth of energy at time $t$.

\section{Pseudospectra and response to external excitations}

The behavior of a non-norm operator depends not solely on the eigenvalues, but on the structure of $\epsilon$ pseudospectra. As discussed in Sec. III, the resolvent norm represents the amplification of response to external forcing. In order to know the response to external excitations, we plot the $\epsilon$ pseudospectra for several sets of typical parameters. Figure 3 shows the $\epsilon$ pseudospectra and the spectrum for axisymmetric disturbances for $n=0.8$ and 0.2 at $\overline{\mathrm{Re}}=3000$ and $k=1.0$. For pipe Poiseuille flow of a Newtonian fluid, the eigenvalues locate on three main branches, i.e., $A$ branch, $P$ branch, and $S$ branch [10]. A modes, which have their largest variation close to the wall, have rather small phase velocities. Whereas, the $P$ modes, which have their maxima close to the center of the channel, have much higher phase speeds. The $S$ modes, which are highly damped, have a phase speed that is nearly equal to $2 / 3$. We have computed the eigenvalue problem for a wide range of Reynolds numbers for shear-thinning cases. The computational results show that all the eigenvalues are stable. As shown in Fig. 3 for non-Newtonian cases, the structures of the pseudospectra and the spectrum are similar to the Newtonian case. Comparing Fig. 3(a) with Fig. 3(b), it is found that with the increase of shear thinning the spectrum becomes more continuous in the $S, P$, and $A$ branches. Consider the $\epsilon$ pseudospectrum for $\epsilon=10^{-5}$, whose boundary is the inner contour in Fig. 3(a). This set covers the vicinity of the intersection of branches. As shown in Fig. 3(b), for $n=0.2$ the $\epsilon$ pseudospectrum for $\epsilon=10^{-5}$ extends to a much larger region, i.e., it covers the intersection of branches and the $S$ branch. This result indicates that the eigenvalues 
(a)

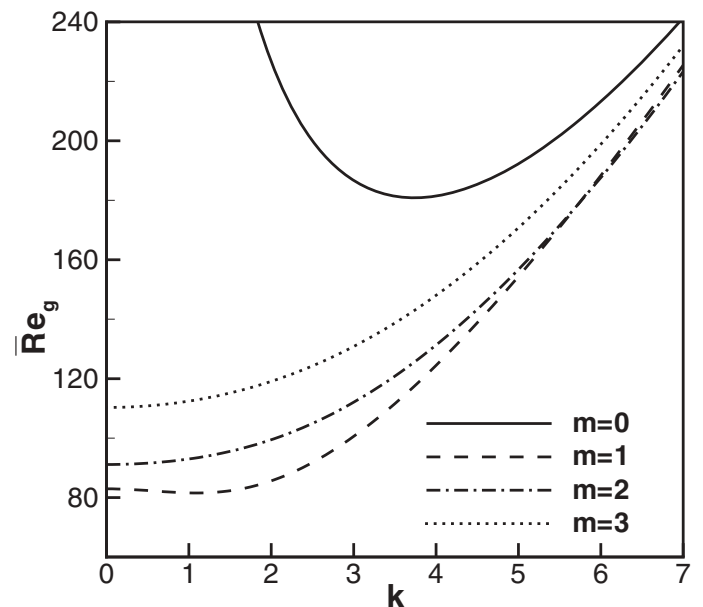

(c)

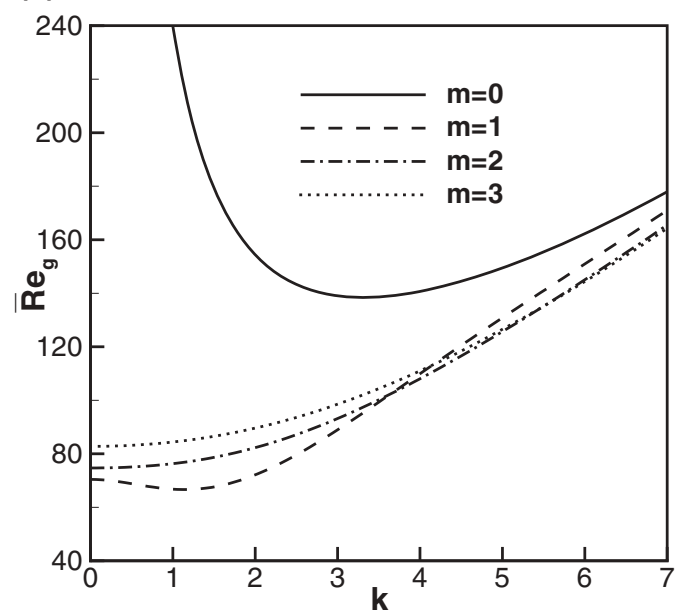

(b)

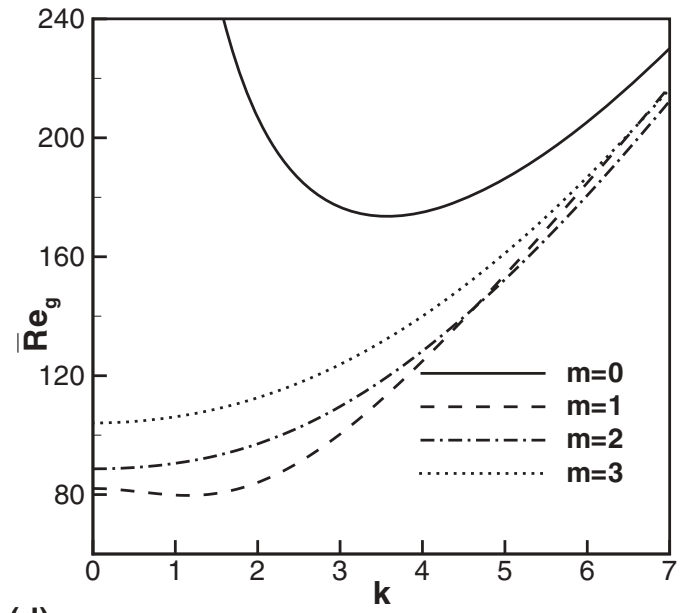

(d)

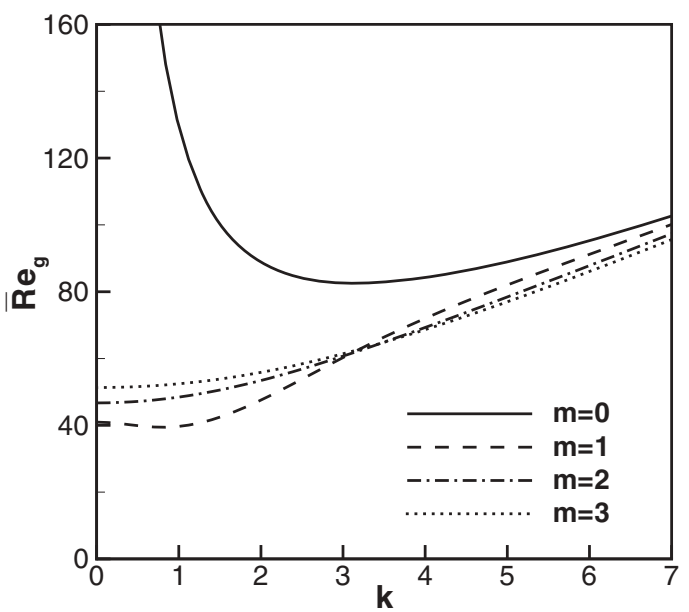

FIG. 2. The curves of the energy Reynolds number $\overline{\operatorname{Re}}_{g}$ versus the streamwise wave number $k$ for various azimuthal wave numbers. (a) $n=1$, (b) $n=0.8$, (c) $n=0.5$, (d) $n=0.3$. The dimensionless time constant $\lambda=10$.

(a)

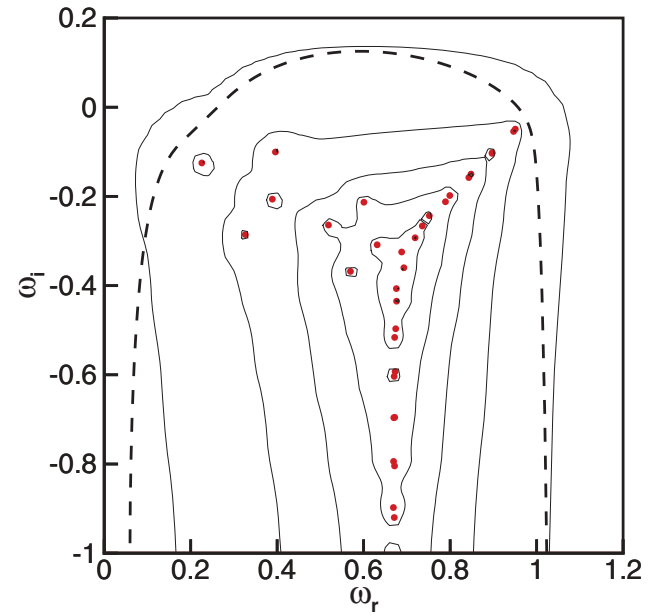

(b)

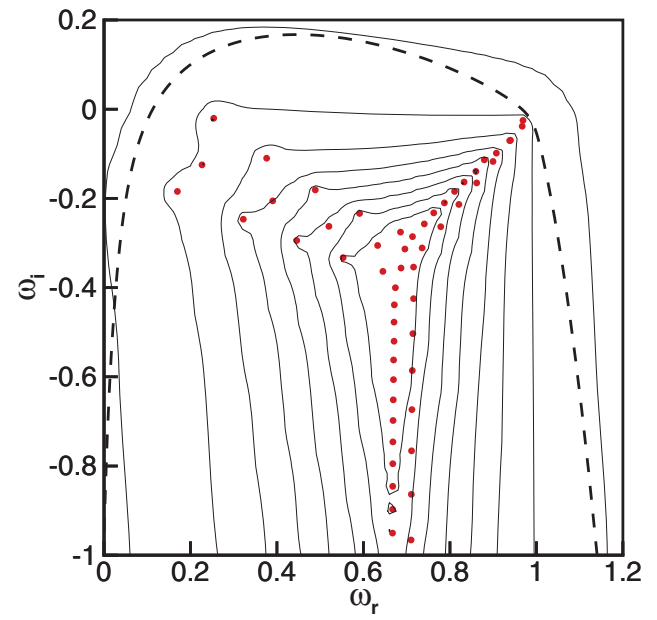

FIG. 3. (Color online) Pseudospectra for the pipe Poiseuille flow with $k=1.0, m=0$ at $\overline{\operatorname{Re}}=3000$ for (a) $n=0.8$, (b) $n=0.2$. Dotted line: eigenvalues; dashed line : boundary of the numerical range; solid lines: contours from outermost to innermost representing levels from $\epsilon=10^{-1}$ to $10^{-i}$. The dimensionless time constant $\lambda=10$. 
(a)

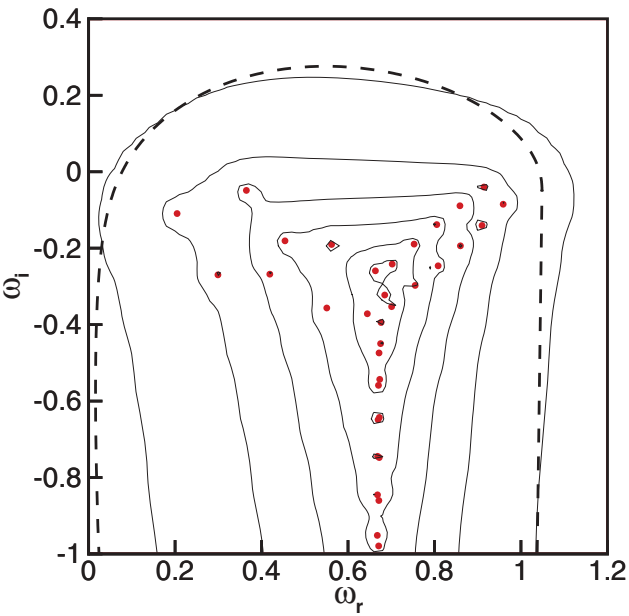

(c)

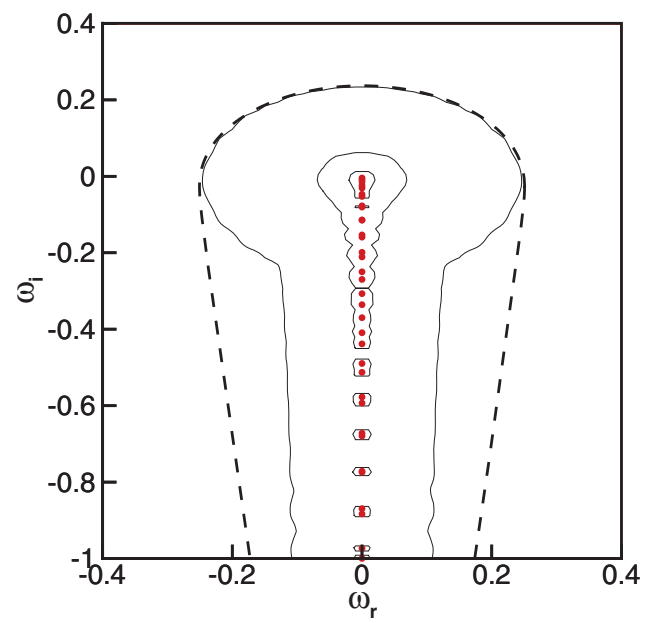

(b)

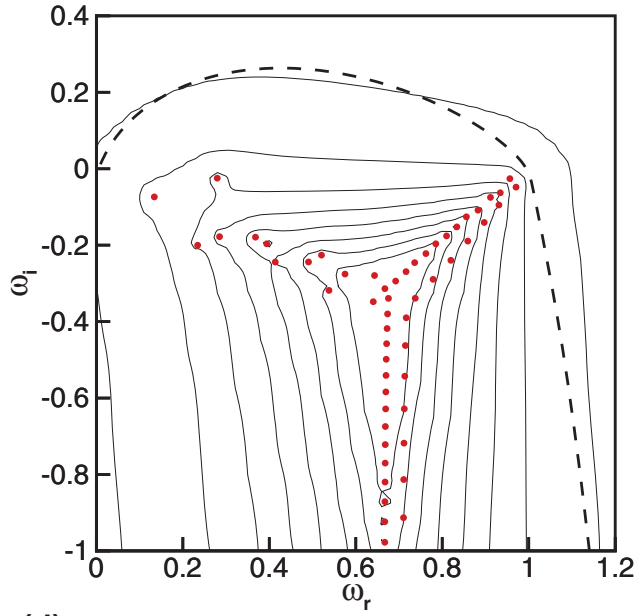

(d)

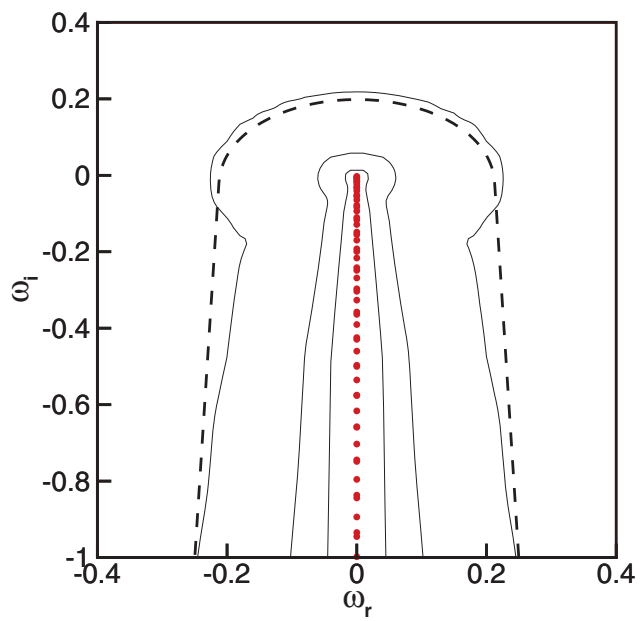

FIG. 4. (Color online) Pseudospectra for the plane Couette flow with $k=1.0, m=1$ at $\overline{\operatorname{Re}}=3000$ for (a) $n=0.8$, (b) $n=0.2$. Pseudospectra for the plane Couette flow with $k=0, m=1$ at $\overline{\mathrm{Re}}=3000$ for (c) $n=0.8$, (d) $n=0.2$. Dotted line: eigenvalues; dashed line: boundary of the numerical range; solid lines: contours from outermost to innermost representing levels from $\epsilon=10^{-1}$ to $10^{-i}$. The dimensionless time constant $\lambda=10$.

near the intersection are highly sensitive to perturbations, and the sensibility increases with the increase of shear thinning. In Fig. 3(b), we found it interesting that for $n=0.2$, the $S$ branch consists of spectra locating in two straight lines because the eigenvalues of the Squire mode depart from the Orr-Sommerfeld mode [10] in the $S$ branch.

In Fig. 4, we plot the spectrum and pseudospectra for nonaxisymmetric cases with the azimuthal wave number $m=1$. In Figs. 4(a) and 4(b), the disturbance is streamwise dependent with the streamwise wave number $k=1.0$. The structures of spectrum and pseudospectra in Figs. 4(a) and 4(b) are very similar to the axisymmetric cases in Figs. 3(a) and 3(b). This means the effect of shear thinning is qualitatively the same for both the axisymmetric and nonaxisymmetric cases if the disturbance is streamwise dependent.

Figures 4(c) and 4(d) show the $\epsilon$ pseudospectrum and spectrum for streamwise uniform disturbance for $n=0.8$ and 0.2 . In these two figures, the structures of the pseudospectrum and eigenvalues are qualitatively similar to the Squire mode of pipe Poiseuille flow of the Newtonian case. Comparing these two figures, we found that with the increase of shear thinning the spectrum becomes more continuous. In Figs. 3 and 4 for the axisymmetic and nonaxisymmetic cases, all the curves of the numerical range show that the numerical abscissa is positive. This means that at the initial time the energy of the disturbance is growing. Comparing Figs. 4(a) with 4(b) and 4(c) with 4(d), it is shown that the numerical abscissa is insensitive to the change of shear thinning. This is because transient growth is an inviscid mechanism. However, the effect of shear thinning becomes dominant in the viscous stage.

In the complex $\omega$ plane, we are particularly interested in the resonance of the real axis because it corresponds to external excitations at real frequencies. In Fig. 5, we plot the maximum response to spatially harmonic forcing at a wide range of frequencies for several typical cases at $\overline{\mathrm{Re}}=3000$. In Fig. 5(a), the curves of the response of disturbance in the form 
(a)

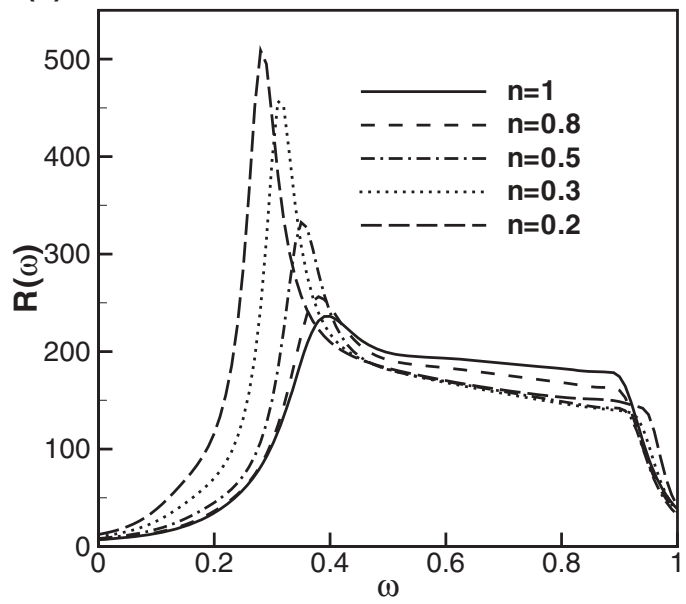

(b)

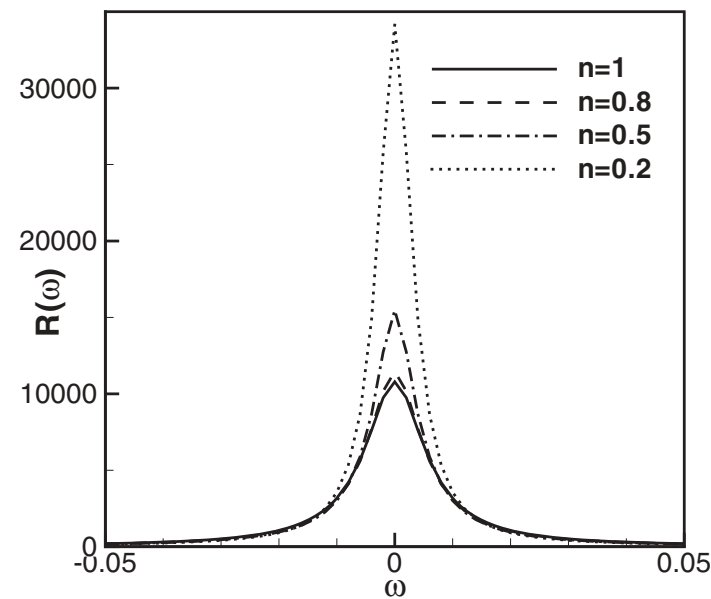

(c)

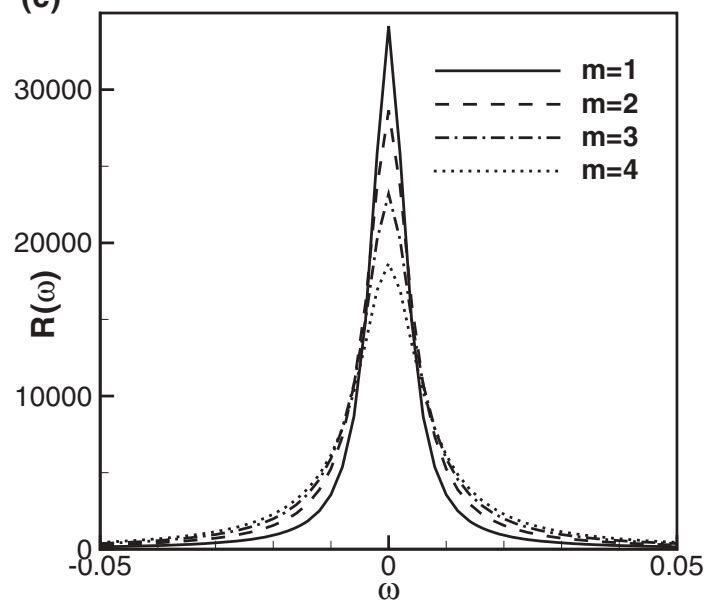

FIG. 5. The curves of the maximum response $\mathcal{R}(\omega)$ to the harmonic external forcing versus the real frequency $\omega$ at $\overline{\operatorname{Re}}=3000$. (a) $k=1.0$, $m=1$ for various $n$, (b) $k=0, m=1$ for various $n$, (c) $n=0.2, k=0$ for various $m$. The dimensionless time constant $\lambda=10$.

of oblique waves with $k=1.0$ and $m=1$ are presented for various non-Newtonian index $n$. The structures of the response curves are similar for various $n$. In Fig. 5(a), all the curves have shown that external forcing is significantly amplified in a limited band of frequency. The amplification at the left (right) side of the band is attributed to the resonance of external forcing and the wall (center) mode. In the inner region of the frequency band, external forcing is significantly amplified because the modes in $S$ branch is very sensitive to disturbance. As shown in Fig. 5(a), with the increase of shear thinning the amplification of the response increases and the frequency corresponding to the maximal response shifts to a lower value.

In Fig. 5(b), the curves of the response of streamwise uninform disturbances with $m=1$ are plot for various nonNewtonian index $n$. In Fig. 5(b) for streamwise independent disturbances, the disturbance is mainly amplified in the low frequency range and decreases significantly in the high frequency range. The most amplified response is achieved at the frequency $\omega=0$. The maximal amplifications are of the order of $10^{4}$ which are much higher than those of oblique waves in Fig. 5(a). In Fig. 5(b), with the increase of shear thinning the amplification increases. In Fig. 5(c), the curves of streamwise uniform disturbances with $n=0.2$ are plotted for various azimuthal wave numbers. In this figure, the structures of the curves are qualitatively similar. It is found that the disturbances with the azimuthal wave number $m=1$ are amplified more than other modes with $m>1$.

In order to know more about the physical mechanism of the response to external forcing, we present the spatial structures of the optimal input of harmonic forcing and the output for $n=0.2$ at $\overline{\mathrm{Re}}=3000$ in Fig. 6 . The computational results show that the optimal response is in the form of streamwise independent streaks with nonzero azimuthal wave numbers. As the optimal frequency is $\omega=0$, only plots of input and output are needed. The computational results show that for the optimal input the radical and azimuthal velocity components have most of the perturbation kinetic energy. For the optimal output, the streamwise component has most of kinetic energy. The flow fields of optimal input and output are presented for $n=0.2$ and $\overline{\mathrm{Re}}=3000$ in Figs. 6(a) and 6(b) for $m=1$ and in Figs. 6(c) and 6(d) for $m=2$. We also compute the flow fields for various $n$, the structures of the flow fields are similar to those in Fig. 6. 
(a)

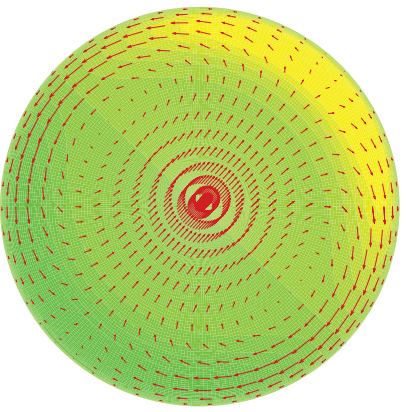

(c)

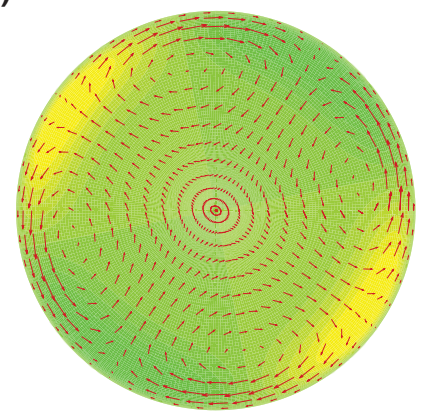

(b)

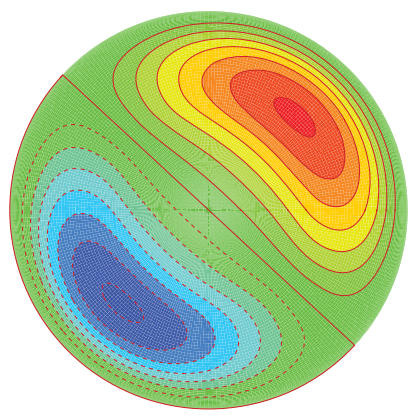

(d)

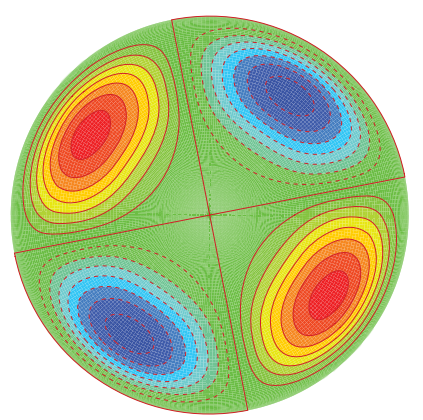

FIG. 6. (Color online) Cross-stream $(r-\theta)$ view of the optimal flow fields of the response to external excitations at $\overline{\mathrm{Re}}=3000, k=0$ for $n=0.2$. The velocity components of $u$ and $v$ of optimal input: (a) $m=1$, (c) $m=2$. Isolines of the velocity component $w$ of optimal output: (b) $m=1$, (d) $m=2$. The dimensionless time constant $\lambda=10$.

\section{Transient growth and optimal response to initial conditions}

In this subsection, we will study the transient energy growth and the response of initial conditions. We can define the energy growth maximized over time as

$$
G_{\max }=G\left(t_{\mathrm{opt}}\right)=\max G(t), \quad t \geqslant 0 .
$$

In order to know the effect of shear thinning on the transient energy growth, we plot in Fig. 7 the curves of energy growth function $G(t)$ for streamwise independent disturbances with various non-Newtonian index $n$ and time constant $\lambda$. In Fig. 7(a), we fixed $\lambda$ and change the value of $n$. As shown in Fig. 7, each curve experiences a substantial energy growth and then gradually decreases with time. Being similar to plane channel flows, for streamwise disturbances the "lift-up" effect is responsible for the energy growth $[13,14]$. It is found that for large values of $n$ the transient energy growth is insensitive to the change of $n$. However, as $n$ becomes small the transient energy growth significantly increases with the increase of shear thinning. In Fig. 7(b), we fixed $n$ to be 0.5 and plot the transient energy growth function for various time growth constant $\lambda$. In all the curves in Fig. 7(b), the curve of $\lambda=1$ has the lowest maximum transient growth. For $\lambda>1$, it is obvious that with the increase of $\lambda$ the transient energy growth increases.

In Fig. 8, we plot the curves of the transient energy growth function with various azimuthal wave numbers $m$ for different $n$. In Fig. 8(a) for $n=0.8$, the streamwise uniform disturbance

\section{(a)}

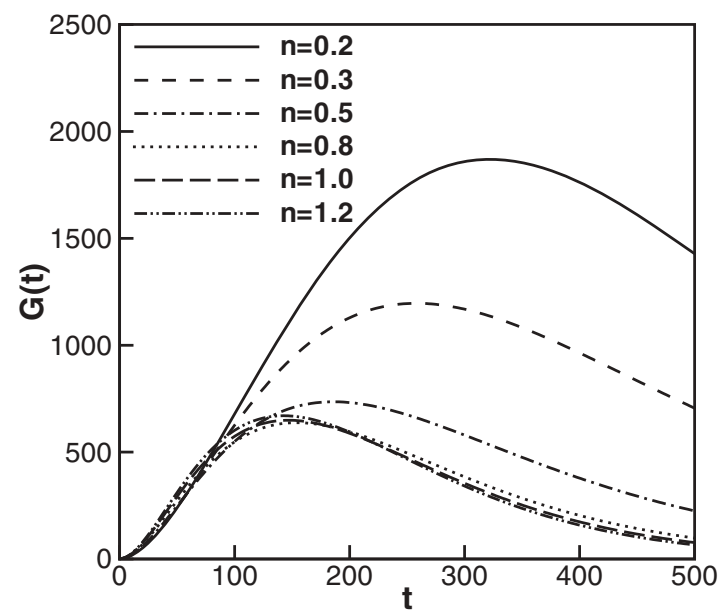

(b)

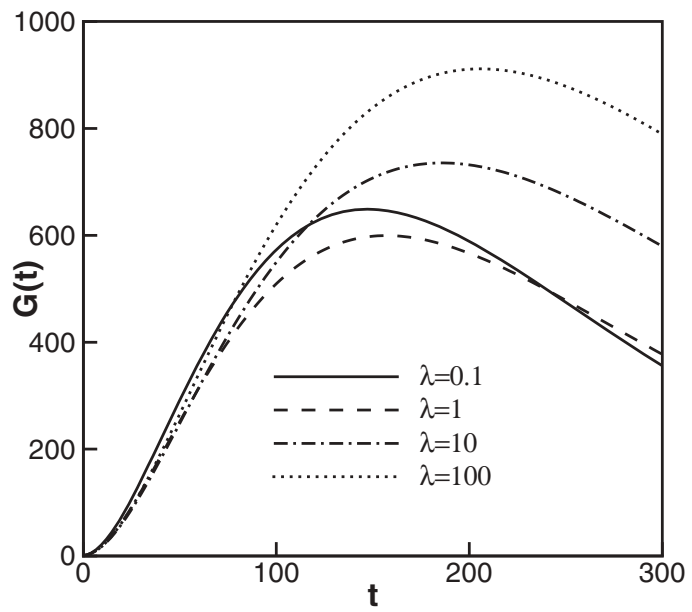

FIG. 7. The curves of transient energy growth function $G(t)$ versus time $t$ for various azimuthal wave numbers at $\overline{\operatorname{Re}}=3000$. (a) $m=1$, $k=0$ with $\lambda=10$ for various $n$, (b) $m=1, k=0$ with $n=0.5$ for various $\lambda$. 
(a)

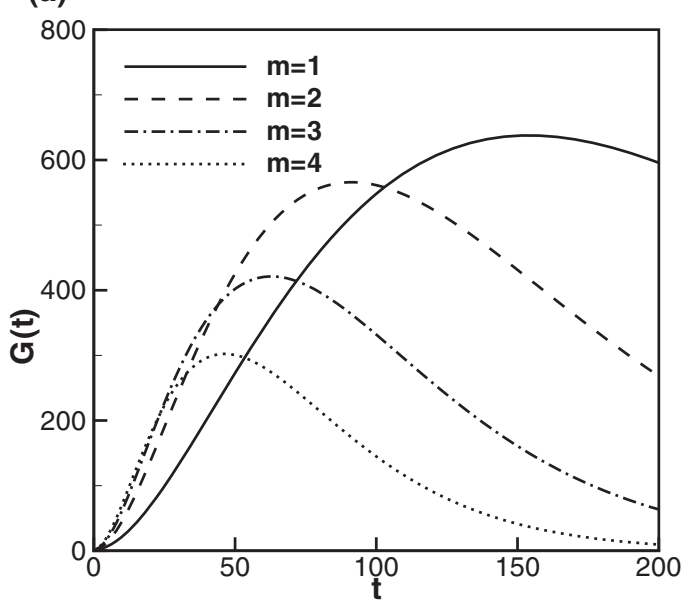

(c)

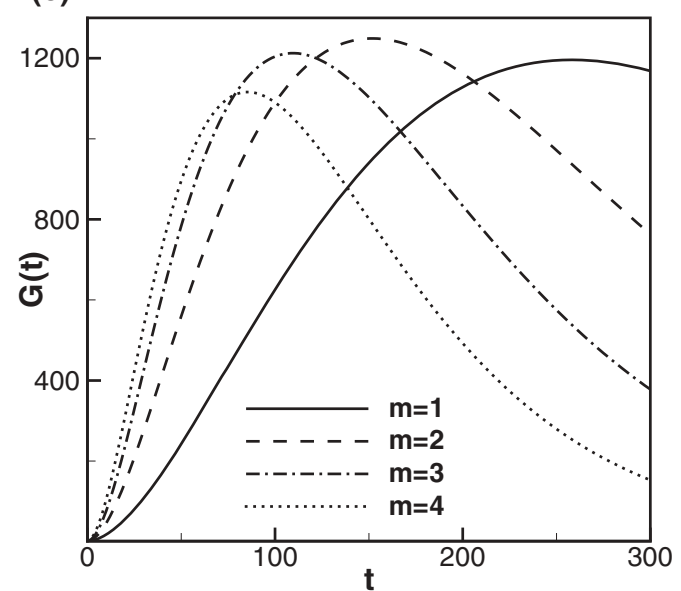

(b)

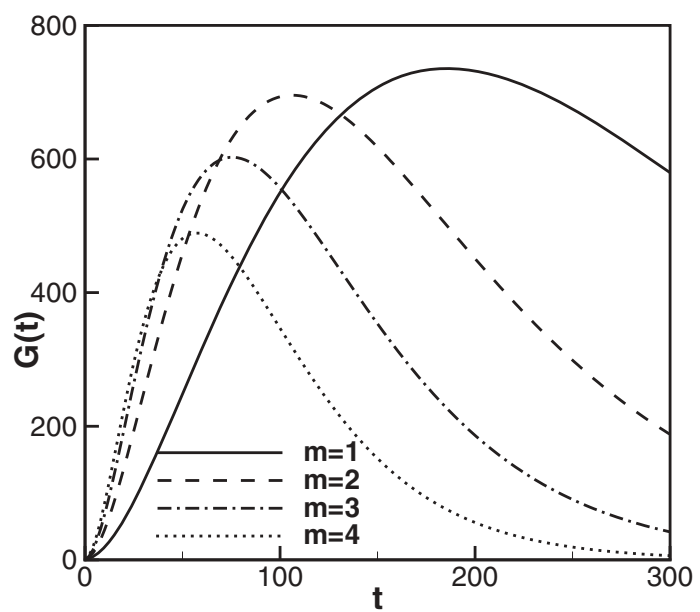

(d)

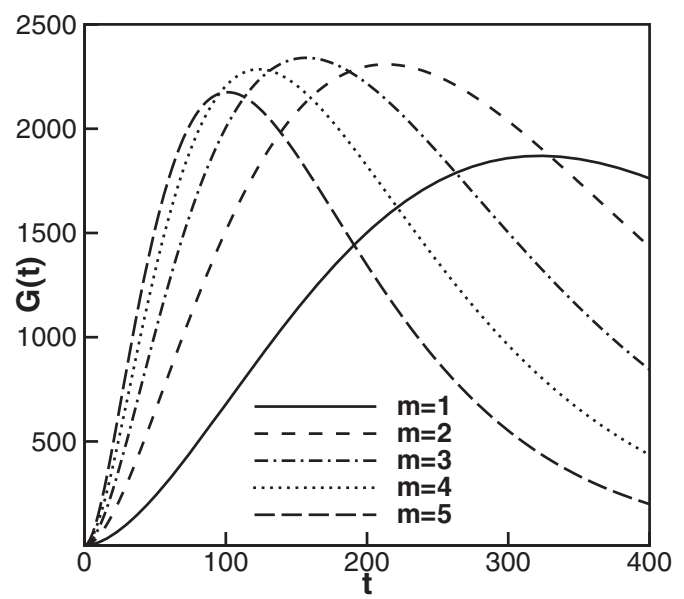

FIG. 8. The curves of transient energy growth function $G(t)$ versus time $t$ for various azimuthal wave numbers at $\overline{\operatorname{Re}}=3000$. (a) $n=0.8$, $k=0$, (b) $n=0.5, k=0$, (c) $n=0.3, k=0$, (d) $n=0.2, k=0$. The dimensionless time constant $\lambda=10$.

with $m=1$ has a higher optimal energy growth than those with $m>1$. However, at the initial stage, the energy growth rate of the disturbance with $m=1$ is lower than those with $m>1$. As $n$ decreases to 0.5, as shown in Fig. 8(b), the disturbance with $m=1$ also has a higher optimal energy growth than other modes. However, it is found that for $n=0.5$ the difference between $G_{\max }$ of $m=1$ and $m=2$ is less than that for 0.8 , as shown in Fig. 8(a). As $n$ decreases further to 0.3, as shown in Fig. 8(c), it is found that the disturbance with $m=2$ has the largest optimal transient energy growth. The maximum transient energy growth of $m=3$ is slightly lower than that of $m=2$ and higher than that of $m=1$. As shown in Fig. 8(d) for $n=0.2$, we found it interesting that the disturbance with $m=3$ has the highest optimal energy growth. In this figure, the energy growths of $m=2,3,4,5$ are obviously higher than that of $m=1$. The dependence of the amplification of disturbance on the azimuthal wave number $m$ for the initial problem is significantly different from that for the problem of response to external excitations, in which the disturbance of $m=1$ always has the largest amplification of response.

In order to know the effect of shear thinning on the structure of disturbance with optimal energy growth, we plot in Fig. 9 the flow fields of the optimal disturbance at the initial time $t=0$ and $t=t_{\mathrm{opt}}$ with the azimuthal wave number $m=1$ for $n=0.8$ and 0.2 . At the initial time $t=0$ and optimal time $t=t_{\mathrm{opt}}$, the flow fields are characterized by two counter-rotating vortices and streaks. The flow structures of the initial problem in Fig. 9 is very similar to that of the input-output problem in Fig. 6. Comparing the structure of the optimal flow fields in Fig. 9(b) for $n=0.8$ with that in Fig. 9(d) for $n=0.2$, it is shown that with the increase of shear thinning, the centers of the streaks shift from the center region to the wall region. Esmael and Nouar [21] studied the linear stability of Hagen-Poiseuille flow of Herschel-Bulkley fluid to infinitesimal disturbances. The streaky structure in Fig. 9(d) is very similar to that in Ref. [21] for a shear-thinning fluid with $n=0.5$. We display in Fig. 10 the optimal flow field for the modes with the azimuthal wave number $m=2$ and 3 for different values of $n$. In these figures, it is obvious that with the decrease of $n$, the centers of the streaks shift towards the wall region. Moreover, a stagnant region exists near the center of pipe for the strongly shear-thinning case as shown in Figs. 10(b), 10(e), and 10(f). In order to know the growth mechanism of streamwise uniform streaks in Hagen-Poiseuille flow, it is helpful to recall the linearized momentum equation for the streamwise velocity component 
(a)

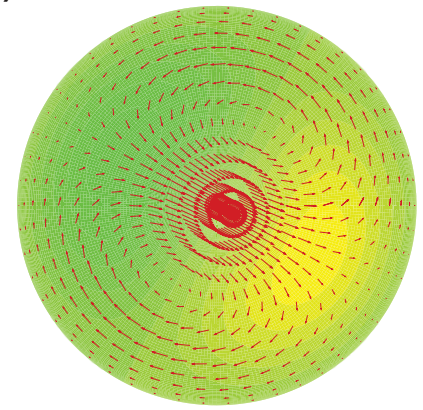

(c)

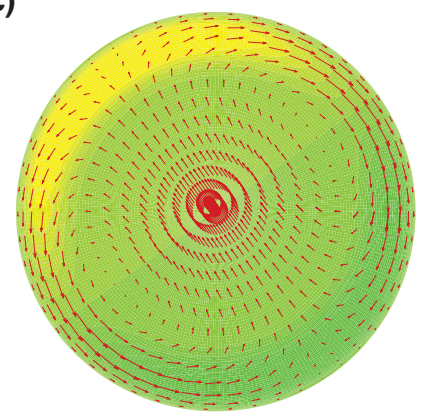

(b)

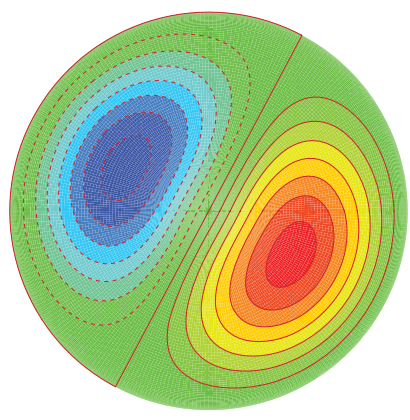

(d)

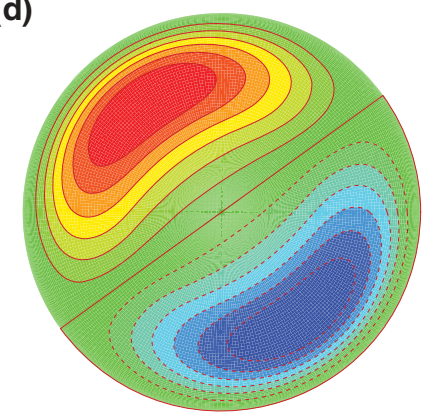

FIG. 9. (Color online) Cross-stream $(r-\theta)$ view of the optimal flow fields of the response to initial conditions at $\overline{\mathrm{Re}}=3000, k=0$. The velocity components of $u$ and $v$ at the initial time: (a) $n=0.8$, (c) $n=0.2$. Isolines of the velocity component $w$ at the optimal time: (b) $n=0.8$, (d) $n=0.2$. The dimensionless time constant $\lambda=10$. $w$ of streamwise uniform disturbances:

$$
\frac{\partial}{\partial t} w=-\frac{d W_{b}}{d r} u .
$$

For inviscid shear flows, the streamwise velocity grows linearly with time for disturbances with no streamwise variation [12]. As shown in Fig. 1(a), with the increase of shear thinning the gradient of $W_{b}$ increases near the wall and the velocity profile flattens in the central region. From Eq. (43) it can be shown that the mean shear near the wall provides more energy to the disturbance because the base velocity gradient becomes larger near the wall with the increase of shear thinning. In the central region, the velocity profile becomes flatter with the increase of shear thinning. The formation of the stagnant region in Figs. 10(b) and 10(d) is due to the absence of the lift-up mechanism in the central region. The result in which the centers of the streaks moving towards the wall with the increase of the shear thinning is mainly due to the modification of the profile of the axial velocity of base state. For plane Poiseuille flow, the spanwise wavelength is about two times the height of the channel. For Hagen-Poiseuille flow, with the increase of shear thinning the axial size of the optimal streaks decreases. It is natural that the azimuthal size of the streak should decrease to match the axial size. So, the azimuthal wave number may be greater than 1 for strongly shear-thinning cases as shown in Figs. 8(c) and 8(d).

We have examined the transient energy growth of the streamwise independent disturbance. In order to know the effect of shear thinning on the transient energy growth of a spatially stochastic disturbance, it is helpful to study the energy growth of streamwise dependent disturbances. Figure 11 displays the curves of $G_{\max }$ versus the streamwise wave number $k$ with various $m$ at $\overline{\mathrm{Re}}=3000$ for $n=0.8$ and 0.2 . As shown in these two figures, the maximum energy growth of each curve is realized at the streamwise wave number $k=0$. For $n=0.8$ in Fig. 11(a), the disturbance with $m=1$ has the largest transient energy growth. With the increase of streamwise wave number, $G_{\max }$ of $m=1$ decreases more than other modes with $m>1$. In the shortwave range, the disturbance with $m=3$ has the largest $G_{\max }$. For $n=0.2$ in Fig. 11(b), it is found that the disturbance with $m=3$ has the largest $G_{\max }$. Moreover, the energy growth of $m=2-6$ are all significantly higher than that of $m=1$. In the shortwave range, the mode of $m=6$ has the largest transient energy growth.

For Hagen-Poiseuille flow of a Newtonian fluid, the dependence of the optimal growth on the Reynolds number is known to scale with the square of the Reynolds number. In the present study, in most of the cases the Reynolds number is kept fixed at 3000 . One might wonder whether there is a similar relation between the optimal growth and the Reynolds number for shear-thinning cases as that for the Newtonian case, moreover, whether the azimuthal wave number of the optimal growth changes with the increase of the Reynolds number.

In Fig. 12, the optimal growth over the azimuthal wave number $m$ is displayed for two typical shear-thinning cases of $n=0.5$ and 0.2 at $\overline{\mathrm{Re}}=1000$ and 5000. For $n=0.5$ and 0.2 , the azimuthal wave numbers are $m=1$ and 3 . These values are identical to that for $\overline{\mathrm{Re}}=3000$ as shown in Figs. 8(b) and 8(d). For $n=0.5$ and 0.2 , the curves of $\overline{\operatorname{Re}}=1000$ coincide well with that of $\overline{\mathrm{Re}}=5000$. This result shows that the azimuthal wave number of the optimal growth does not change with the Reynolds number.

In Figs. 13(a) and 13(b), the ratio of optimal growth $G_{\max }$ and the square of the Reynolds number $\overline{\mathrm{Re}}^{2}$ and the ratio of $t_{\mathrm{opt}}$ and $\overline{\mathrm{Re}}$ are plotted at various non-Newtonian index $n$ for $m=1,2$, and 3. In Fig. 13(a), the curves of $\overline{\mathrm{Re}}=1000$ and 
(a)

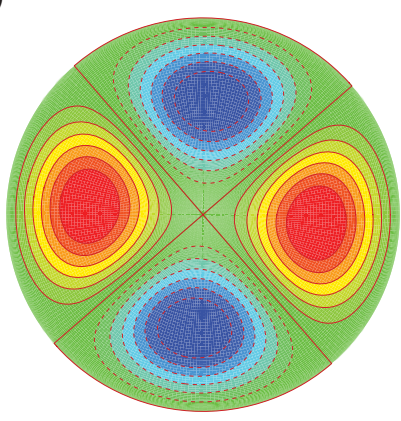

(c)

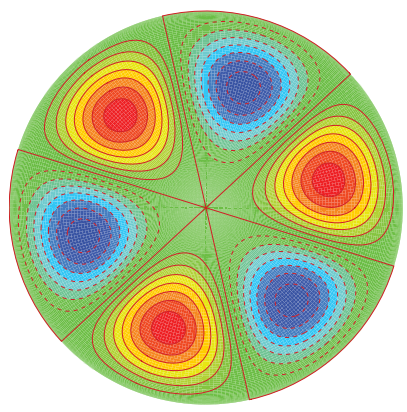

(e)

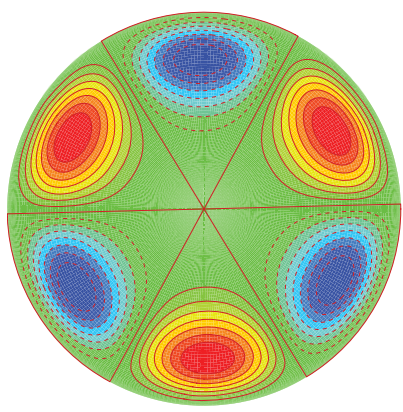

(b)

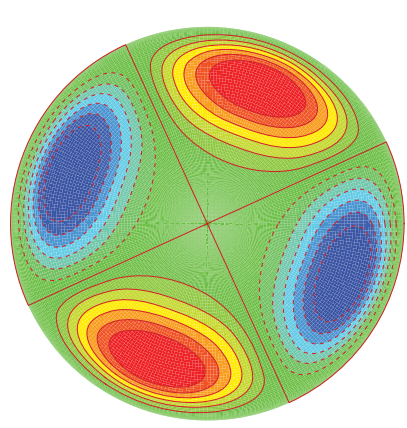

(d)

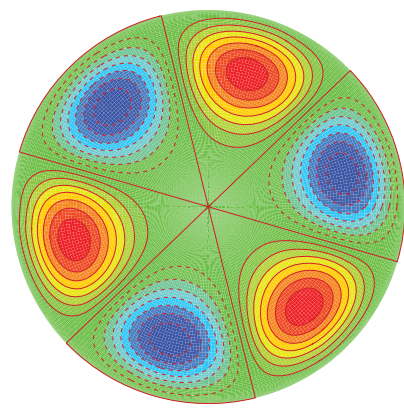

(f)

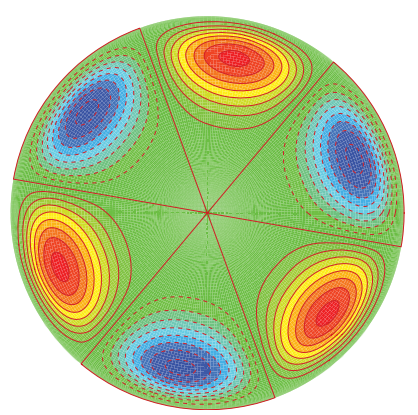

FIG. 10. (Color online) Cross-stream $(r-\theta)$ view of the optimal flow fields of the response to initial conditions at $\overline{\mathrm{Re}}=3000, k=0$. Isolines of the velocity component $w$ at the optimal time (a) $m=2, n=0.8$, (b) $m=2, n=0.5$, (c) $m=$ $3, n=0.8$, (d) $m=3, n=0.5$, (e) $m=3, n=$ 0.3 , (f) $m=3, n=0.2$. The dimensionless time constant $\lambda=10$. (a)

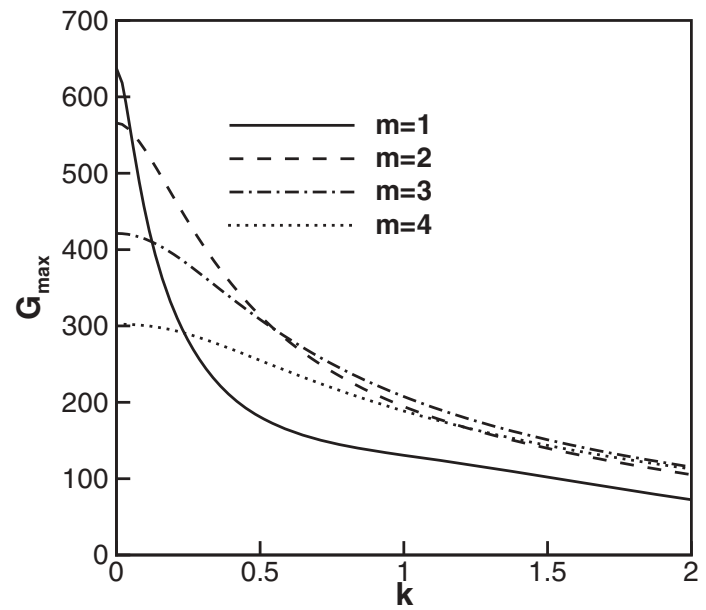

(b)

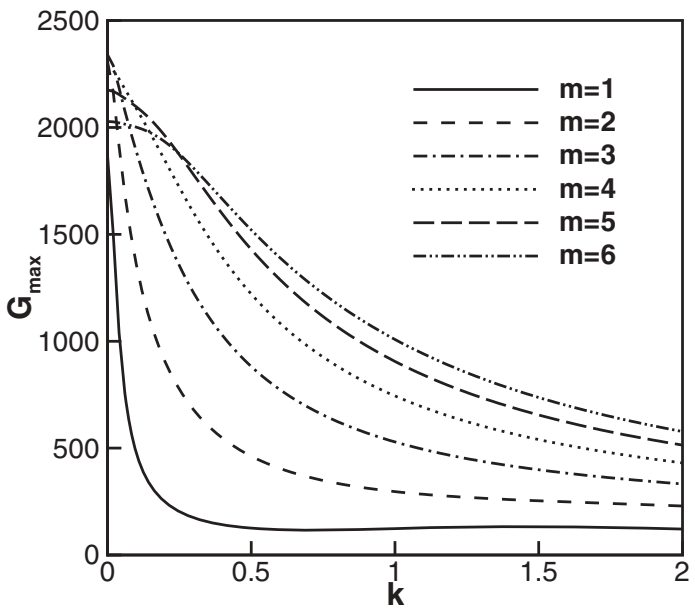

FIG. 11. The maximum transient energy growth $G_{\max }$ versus the streamwise wave number $k$ at $\overline{\operatorname{Re}}=3000$. (a) $n=0.8$, (b) $n=0.2$. The dimensionless time constant $\lambda=10$. 


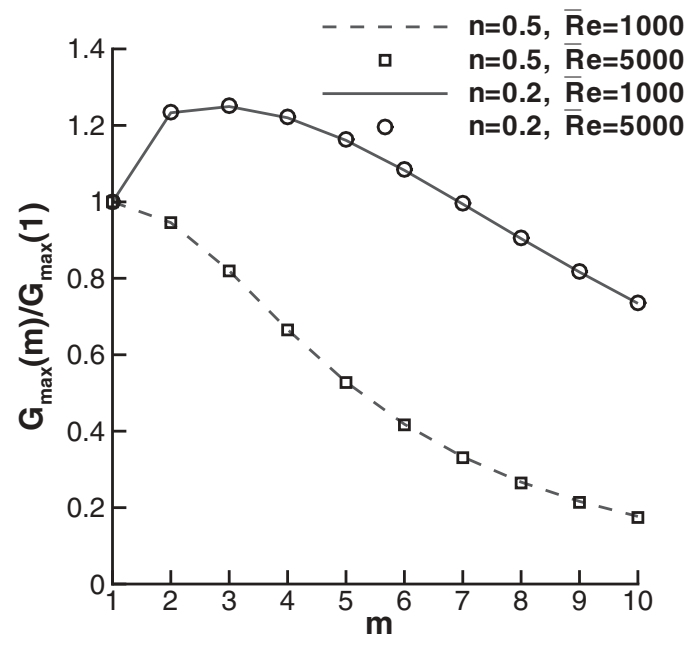

FIG. 12. The optimal transient growth $G_{\max }$ at various azimuthal wave numbers $m$. The dimensionless time constant $\lambda=10$.

5000 coincide. This result implies a quadratic scaling of the optimal growth with the Reynolds number and as $\overline{\operatorname{Re}} \rightarrow \infty$ for shear-thinning cases. In Fig. 13(b), the curves of the ratio of $t_{\mathrm{opt}}$ and $\overline{\mathrm{Re}}$ show that the optimal time $t_{\mathrm{opt}}$ is of order $\overline{\mathrm{Re}}$ for all modes with various azimuthal wave numbers. In Fig. 13(a), for fixed Reynolds number $\overline{\operatorname{Re}}$ the optimal growth of $m=1$ is insensitive to shear thinning as $n$ is large. For $m=2$ and 3, for large values of $n$, the optimal growth gradually increases with the decrease of $n$. In the range of $n<0.7$, the optimal growth of each $m$ significantly increases with the shear-thinning effect. In Fig. 13(b), for fixed $\overline{\mathrm{Re}}$, the optimal time $t_{\mathrm{opt}}$ increases with the increase of shear thinning.

The definition of a Reynolds number in a flow in which the viscosity of the fluid varies in space is not unique as many viscosity scales can be chosen. To assess how shear thinning affects the optimal growth rate, it is important to employ an appropriate viscosity scale in the definition of the Reynolds number. In Fig. 14(a) a global view of the effect of shear thinning on the optimal transient growth is provided for the
Reynolds number based on the zero-shear-rate viscosity $\hat{\mu}_{0}$. Figure 14(a) shows that shear thinning significantly enhances the optimal energy growth for fixed Re. In Fig. 14(b), the optimal time increases with the increase of shear thinning. These results are qualitatively similar to that for fixed Reynolds numbers by using the viscosity averaged across $z$ as a viscosity scale, as shown in Figs. 13(a) and 13(b). Comparing Fig. 14(a) with Fig. 13(a), it is found that the enhancement of the optimal growth by shear thinning is overestimated by using the zeroshear-rate viscosity as a viscosity scale.

\section{CONCLUSIONS}

In the present paper, we have investigated the linear stability in Hagen-Poiseuille flow of a shear-thinning fluid using the energy method and the nonmodal stability theory. We focus on the effect of shear thinning on the stability of the flow. The critical energy Reynolds number $\overline{\operatorname{Re}}_{g}$ below which there is no energy growth was determined by searching the maximal eigenvalue of $\frac{1}{2}\left(\mathscr{L}-\mathscr{L}^{H}\right)$. We investigated the condition with no energy growth for both axisymmetric and nonaxisymmetric disturbances. The results of energy stability analysis show that each mode with a different azimuthal wave number can experience an energy growth at the initial time. The critical condition is determined by the nonaxisymmetric disturbance with $m=1$ and $1<k<2$. With the increase of the non-Newtonian index $n$, the critical energy Reynolds number decreases.

In the nonmodal stability analysis, we focus on the response to external excitations and initial conditions. The response to external excitations is characterized using the $\epsilon$ pseudospectrum, and the response to initial conditions is described by the energy growth function $G(t)$. For both the Newtonian and non-Newtonian cases, both the axisymmetric and nonaxisymmetric external forcing can be amplified by a rather large factor. However, the amplification of response to the axisymmetric external forcing is much lower than that of the nonaxisymmetric one. For all nonaxisymmetric cases, the disturbance with the azimuthal wave number $m=1$ has (a)

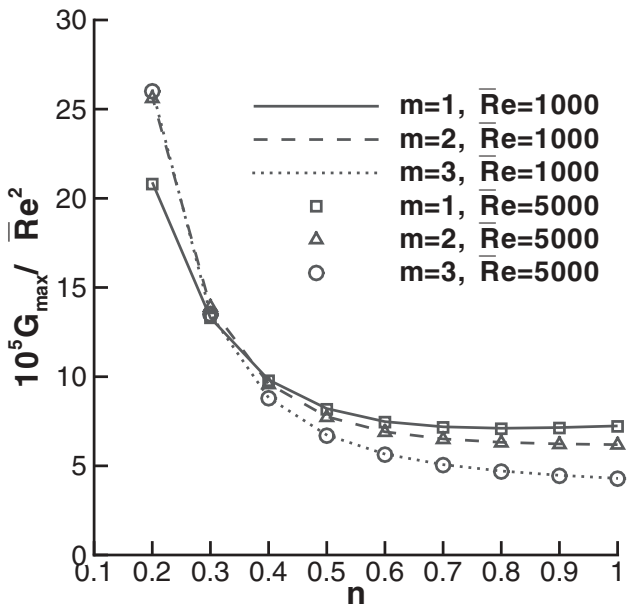

(b)

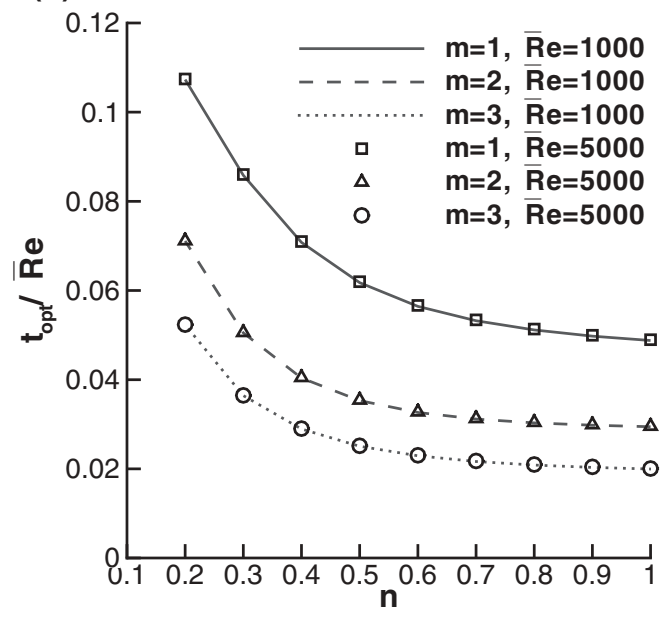

FIG. 13. Effect of $n$ on the optimal transient growth $G_{\max }$ and the optimal time $t_{\text {opt }}$ at fixed Reynolds number $\overline{\operatorname{Re}}=1000$ and 5000 . The dimensionless time constant $\lambda=10$. 

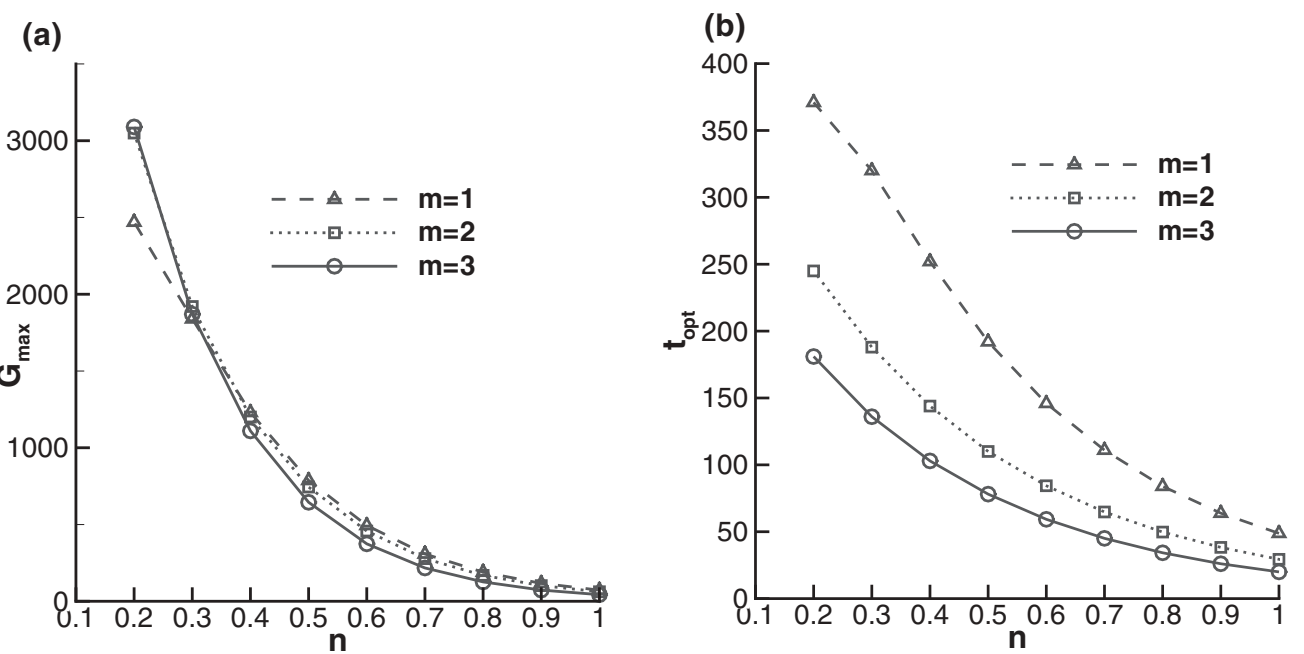

FIG. 14. Effect of $n$ on the transient energy growth $G_{\max }$ and $t_{\max }$ at $\operatorname{Re}=1000$. The dimensionless time constant $\lambda=10$.

the largest amplification. For all values of $m$, the largest amplification is realized at frequency $\omega=0$. We find that the amplification of the response increases with the increase of shear thinning.

We have also studied the transient energy growth of initial conditions. The results show that even though all the eigenvalues of the pipe Poiseuille flow of both Newtonian and non-Newtonian fluids are stable, the disturbance also can be amplified substantially. The optimal transient energy growth is achieved by streamwise independent nonaxisymmetric disturbances. The lift-up effect is responsible for the transient energy growth. In the Newtonian case, the disturbance with $m=1$ has the largest optimal energy growth. However, with the increase of shear thinning, the disturbance with a higher azimuthal wave number may become the optimal disturbance. For example, as $n$ decreases to 0.3 or 0.2 , the azimuthal wave number of the optimal disturbance is 2 or 3 . This result is different from that of the response to external excitation in which the azimuthal of the optimal disturbance is always 1. We have investigated the effect of shear thinning on the structure of the optimal streaks. It is observed that with the increase of shear thinning, the centers of the optimal streaks shift towards the wall region. This result is due to the modification of the profile of the base axial velocity by shear thinning.

To assess how shear thinning affects the optimal growth rate, different viscosity scales are used in the definition of the Reynolds number. We compared the results for the Reynolds number based on the average viscosity and the zero-shear-rate viscosity. For both definitions, the optimal growth increases with the increase of shear thinning. We have investigated the relations between the optimal growth $G_{\max }$ and optimal time $t_{\text {opt }}$ and the Reynolds number $\overline{R e}$ for various degrees of shear thinning $n$ and different azimuthal wave numbers $m$. It is interesting that for each mode of $m$ the optimal growth has a quadratic scaling with the Reynolds number and the optimal time is of the order of $\overline{\mathrm{Re}}$ as $\overline{\mathrm{Re}} \rightarrow \infty$. These relations are qualitatively similar to that for Hagen-Poiseuille flow of the Newtonian case.

\section{ACKNOWLEDGMENTS}

This work was supported by National Natural Science Foundation of China (Grants No. 11102211, No. 50890182, and No. 11072249) and the Knowledge Innovation Program of Chinese Academy of Sciences (KGCX-SW-409).
[1] O. Reynolds, Proc. R. Soc. London 35, 84 (1883).

[2] P. J. Schimid and D. S. Henningson, J. Fluid Mech. 277, 197 (1994).

[3] A. Meseguer and L. N. Trefethen, J. Comput. Phys. 186, 178 (2003).

[4] I. J. Wygnanski and F. H. Champagne, J. Fluid Mech. 59, 281 (1973).

[5] R. R. Kerswell, Nonlinearity 18, R17 (2005).

[6] B. Eckhardt, T. Schneider, B. Hof, and J. Westerweel, Annu. Rev. Fluid Mech. 39, 447 (2007).

[7] P. J. Schmid, Annu. Rev. Fluid Mech. 39, 129 (2007).

[8] L. N. Trefethen, A. E. Trefethen, S. C. Reddy, and T. A. Driscoll, Science 261, 578 (1993).
[9] S. Reddy and D. S. Henningson, J. Fluid Mech. 252, 209 (1993).

[10] P. J. Schmid and D. S. Henningson, Stability and Transition in Shear Flows (Springer-Verlag, New York, 2001).

[11] K. M. Butler and B. F. Farrell, Phys. Fluids A 4, 1637 (1992).

[12] T. Ellingsen and E. Palm, Phys. Fluids 18, 487 (1975).

[13] M. T. Landahl, SIAM J. Appl. Math. 28, 735 (1975).

[14] M. T. Landahl, J. Fluid Mech. 98, 243 (1980).

[15] F. Waleffe, Phys. Fluids 9, 883 (1997).

[16] R. B. Bird, W. E. Stewart, and E. N. Lightfoot, Transport Phenomena (John Wiley \& Sons, New York, 2002).

[17] R. B. Bird, R. C. Armstrong, and O. Hassager, Dynamics of Polymeric Liquid, Fluid Mechanics Vol. I (John Wiley \& Sons, New York, 1977). 
[18] V. Chikkadi, A. Sameen, and R. Govindarajan, Phys. Rev. Lett. 95, 264504 (2005).

[19] C. Nouar, N. Kabouya, J. Dusek, and M. Mamou, J. Fluid Mech. 577, 211 (2007).

[20] R. Liu and Q. S. Liu, J. Fluid Mech. 676, 145 (2011).

[21] A. Esmael and C. Nouar, Phys. Rev. E 77, 057302 (2008).

[22] A. Meseguer and L. N. Trefethen, Numerical Analysis Group Report No. 00/18, Oxford University, Computing Lab, 2000 (unpublished).
[23] M. R. Khorrammi, M. R. Malikm, and R. L. Ash, J. Comput. Phys. 81, 206 (1989).

[24] L. N. Trefethen and M. Embree, Spectra and Pseudospectra: The Behavior of Nonnormal Matrices and Operators (Princeton University Press, Princeton, NJ, 2005).

[25] C. Canuto, M. Y. Hussaini, A. Quarteroni, and T. A. Zang, Spectral Method in Fluid Dynamics (Springer, New York, 1993). 